\title{
Continuation of Point Clouds via Persistence Diagrams
}

\author{
Marcio Gameiro $^{\mathrm{a}}$, Yasuaki Hiraoka ${ }^{\mathrm{b}}$, Ippei Obayashi $^{\mathrm{b}}$ \\ ${ }^{a}$ Instituto de Ciências Matemáticas e de Computação, Universidade de São Paulo, Caixa Postal 668, 13560-970, São \\ Carlos, SP, Brazil \\ ${ }^{b}$ WPI-Advanced Institute for Materials Research (WPI-AIMR), Tohoku University, 2-1-1 Katahira, Aoba-ku, Sendai, \\ 980-8577 Japan
}

\begin{abstract}
In this paper, we present a mathematical and algorithmic framework for the continuation of point clouds by persistence diagrams. A key property used in the method is that the persistence map, which assigns a persistence diagram to a point cloud, is differentiable. This allows us to apply the Newton-Raphson continuation method in this setting. Given an original point cloud $P$, its persistence diagram $D$, and a target persistence diagram $D^{\prime}$, we gradually move from $D$ to $D^{\prime}$, by successively computing intermediate point clouds until we finally find a point cloud $P^{\prime}$ having $D^{\prime}$ as its persistence diagram. Our method can be applied to a wide variety of situations in topological data analysis where it is necessary to solve an inverse problem, from persistence diagrams to point cloud data.
\end{abstract}

Keywords: Point Cloud, Persistent Homology, Persistence Diagram, Continuation

\section{Introduction}

Let $P$ be a finite set of points in $\mathbb{R}^{L}$ given by

$$
P=\left\{u_{i} \in \mathbb{R}^{L} \mid i=1 \ldots, M\right\} .
$$

We call $P$ a point cloud, following the convention in topological data analysis (TDA) [1, 2]. TDA provides us tools to study the "shape" of $P$. Among them, persistent homology [3, 4] is one of the most useful tools, and it is now applied into various practical applications, e.g., amorphous solids [5, 6], proteins [7, and sensor networks [8] (see also [1] and references therein).

Persistent homology can be regarded as a collection of maps, called persistence maps in this paper, from $P$ to a finite set $D_{\ell}$, for $\ell=0,1, \cdots$, in the extended plane $\overline{\mathbb{R}}^{2}=\overline{\mathbb{R}} \times \overline{\mathbb{R}}$, where $\overline{\mathbb{R}}=\mathbb{R} \cup\{\infty\}$. The set $D_{\ell}$ is called persistence diagram and it encodes the $\ell$-dimensional topological features of $P$ with metric information (precise definitions are given in Section 2.3).

Email addresses: gameiro@icmc.usp.br (Marcio Gameiro), hiraoka@wpi-aimr.tohoku.ac.jp (Yasuaki Hiraoka), ippei.obayashi.d8@tohoku.ac.jp (Ippei Obayashi)

Preprint submitted to Elsevier
In many applications, the point cloud $P$ have an intricate "shape" or structure, and, in this situations, persistence diagrams are used to provide the "essential" topological features of $P$. For example, in the papers [5, 6, the authors study hierarchical geometric structures in several amorphous solids. In such a case, $P$ is given by an atomic configuration of an amorphous solid and consists of thousands of points in $\mathbb{R}^{3}$ obtained by molecular dynamics simulations. It is a difficult task to directly study the geometry and physical properties of the amorphous solid from $P$ due to its immense size. Hence, a key observation of their work is that the persistence diagrams of the atomic configurations can capture essential geometric information of the amorphous solids. From this significant property, using persistence diagrams they obtain various physical properties of the solid, such as, finding new hierarchical ring structures, decompositions of first sharp diffraction peaks, mechanical responses, etc.

Figure 11 shows a schematic representation of $D_{1}$ for silica glass, $P$, studied in [5] (this corresponds to Figure 1 in that paper). They show that the presence of curves in $D_{1}$ precisely distinguishes the amorphous state from liquid and crystalline states. It means that the normal direc- 
tions to these curves express characteristic geometric constraints on atomic configurations of amorphous states. Therefore, changing $D_{1} \Rightarrow D_{1}^{\prime}$ along a normal direction (e.g., black to red in Figure 1) and tracing the corresponding deformation $P \Rightarrow P^{\prime}$ in the atomic configurations clarify the geometric origin of rigidity in the amorphous solid, which is currently an important problem in physics and material sciences. For this purpose, we need to solve an inverse problem: given $D_{1}^{\prime}$ find $P^{\prime}$ in some appropriate setting, and this is the main subject of this paper.

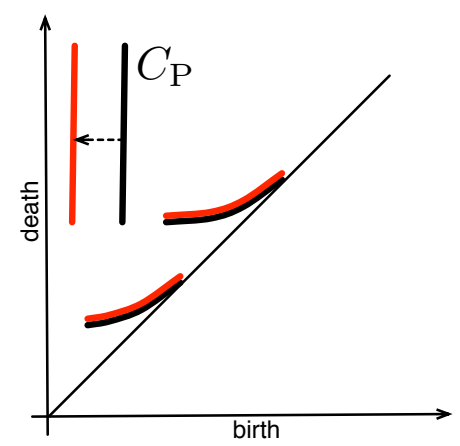

Figure 1: Schematic representation of persistence diagrams. Black: $D_{1}$ for silica glass (see 5 for details). Red: A target persistence diagram $D_{1}^{\prime}$ to study the geometric constraints generating the curve $C_{\mathrm{P}}$

In this paper, we present a mathematical and algorithmic framework for solving inverse problems of persistence diagrams. Our method is based on the continuation method [9, 10, which was originally developed in numerical bifurcation theory of dynamical systems, applied to the setting of persistent homology. More precisely, given a known correspondence between a point cloud $P$ and its persistence diagram $D_{\ell}$, and a target persistence diagram $D_{\ell}^{\prime}$, we develop a method to obtain a point cloud $P^{\prime}$ which have $D_{\ell}^{\prime}$ as its persistence diagram. We first represent the persistence diagrams as points in some Euclidean space and divide the line segment $\overline{D_{\ell} D_{\ell}^{\prime}}$ into small segments $D_{\ell}^{(0)}=D_{\ell}, D_{\ell}^{(1)}, \ldots, D_{\ell}^{(N-1)}, D_{\ell}^{(N)}=D_{\ell}^{\prime}$. Then, we solve an implicit equation defined by the persistence diagram $D_{\ell}^{(i)}$ for each small segment, using the Newton-Raphson method, to obtain a new point cloud $P^{(i)}$ having $D_{\ell}^{(i)}$ as its persistence diagram. By successively applying this procedure, we finally obtain a desired point cloud $P^{\prime}=P^{(N)}$.

We remark that the inverse problem from a persistence diagram $D_{\ell}$ to a point cloud $P$ is not well-posed in general. Namely, it is possible to have multiple point clouds giving the same $D_{\ell}$ (non-uniqueness). Furthermore, the target persistence diagram may not be located in the image of the persistence map (non-existence). Our approach to these issues is as follows: Regarding nonuniqueness, at each step of the continuation we try to find the point cloud closest to the point cloud on the previous step of the continuation. This assigns a minimality condition on the Euclidean norm of the difference for persistence diagrams, and provides the uniqueness property. Furthermore, this minimality condition is reasonable in the practical applications mentioned above, since our input atomic configuration is usually realized as a minimum point of a certain energy landscape, and hence, finding the closest atomic configuration to the minimum point is a natural choice. Regarding non-existence, we take a practical approach. Namely, we try to apply our continuation method and, if the computation is successful we conclude that our target persistence diagram is in the image of the persistence map. If not, we investigate the reason for non-convergence of the Newton-Raphson method, which could be due to non-existence, the presence of zero singular values, etc (see Section 4.5). We note that it is a very challenging mathematical problem to study the image of the persistence map.

This paper is organized as follows. The fundamental concepts, such as simplicial complex models used to represent the point clouds and persistent homology, are introduced in Section 2. In Section 3 local properties of persistence maps, especially differentiability, are studied in detail. Section 4 is the core of the paper and is devoted to developing the continuation method of point clouds using persistence diagrams. In Section 5 we show some computational examples of the proposed method. Finally, in Section 6, we conclude with a list of future improvements to our continuation method.

\section{Simplicial Complex Model and Persistent Homology}

\subsection{Simplicial Complex Models}

Let $V$ be a finite set, and $\Sigma$ be a collection of subsets of $V$. A simplicial complex is defined by a pair $(V, \Sigma)$ satisfying (i) $\{v\} \in \Sigma$ for all $v \in V$ and (ii) $\sigma \in \Sigma$ and $\tau \subset \sigma$ imply $\tau \in \Sigma$. An element $\sigma \in \Sigma$ with $|\sigma|=\ell+1$ is called an $\ell$-simplex. 
Let $P$ be a point cloud 1.1 in $\mathbb{R}^{L}$. For $0<r<\infty$, we refer to the open ball with radius $r$ as an $r$-ball, and denote it, with center $u_{i}$, by

$$
B_{r}\left(u_{i}\right)=\left\{x \in \mathbb{R}^{L} \mid\left\|x-u_{i}\right\|<r\right\} .
$$

The Vietoris-Rips complex $\operatorname{VR}(P, r)$ of $P$ with radius $r$ is defined as the simplicial complex $(P, \Sigma)$ where the set $\Sigma$ of simplices is determined by

$$
\sigma \in \Sigma \Longleftrightarrow B_{r}\left(u_{s}\right) \cap B_{r}\left(u_{t}\right) \neq \varnothing, \quad \forall u_{s}, u_{t} \in \sigma .
$$

The definition of the Vietoris-Rips complex depends only on the distances of all pairs in $P$. Hence the Vietoris-Rips complex has an advantage that it is computable even if $L$ is large.

The alpha complex $\operatorname{Alp}(P, r)$ [2, 11] is another simplicial complex model of $P$ defined by using the set of $r$-balls $B_{r}\left(u_{i}\right)$. A significant property of the alpha complex is the homotopy equivalence

$$
\bigcup_{i=1}^{M} B_{r}\left(u_{i}\right) \simeq|\operatorname{Alp}(P, r)|,
$$

where $|\operatorname{Alp}(P, r)|$ is a geometric realization of $\operatorname{Alp}(P, r)$. Because of this property, the alpha complex is widely used in practical applications to analyze topological features in $P$. We note that the Vietoris-Rips complex does not satisfy this property in general.

Fast software for computing alpha complexes in dimensions $L=2,3$ is available, e.g., [12]. In this paper, we use alpha complexes for $L=3$, while the case $L=2$ can be similarly treated.

For $0<\alpha<\infty$, an $\alpha$-ball $B$ is called $P$-empty if $B \cap P=\varnothing$. For $\ell=0,1,2,3$, let $\operatorname{Del}_{\ell}(P)$ be the set of $\ell$-simplices $\sigma \subset P$ such that there exists an $P$-empty open ball $B$ with $\partial B \cap P=\sigma$. The $D e$ launay triangulation $\operatorname{Del}(P)$ of $P$ is the simplicial complex whose simplices are given by $\operatorname{Del}_{\ell}(P)$ for $\ell=0,1,2,3$.

The three dimensional alpha complex is defined as a subcomplex of the Delaunay triangulation $\operatorname{Del}(P)$. For each $\sigma \in \operatorname{Del}_{\ell}(P)$, let $B_{\sigma}$ be the smallest open ball with $\sigma \subset \partial B_{\sigma}$, and $\rho_{\sigma}$ be the radius of $B_{\sigma}$. Let us define $G_{0, \alpha}=P$, and $G_{\ell, \alpha}$ to be the set of $\ell$-simplices $\sigma \in \operatorname{Del}_{\ell}(P)$ such that $B_{\sigma}$ is $P$-empty and $\rho_{\sigma}<\alpha$. A simplex in $\cup_{\alpha} G_{\ell, \alpha}$ is called an attaching $\ell$-simplex. The alpha complex $\operatorname{Alp}(P, \alpha)$ is defined as the simplicial complex whose simplices are given by $G_{\ell, \alpha}$ and their faces for $\ell=0,1,2,3$. From this definition, the alpha complex $\operatorname{Alp}(P, \alpha)$ is a subcomplex of the
Delaunay triangulation $\operatorname{Del}(P)$, and we have that $\operatorname{Del}(P)=\bigcup_{\alpha} \operatorname{Alp}(P, \alpha)$.

We note that both simplicial complex models define filtrations of finite type

$$
\begin{aligned}
\operatorname{VR}(P) & =(\operatorname{VR}(P, r))_{r \in \mathbb{R}_{\geq 0}}, \\
\operatorname{Alp}(P) & =(\operatorname{Alp}(P, r))_{r \in \mathbb{R}_{\geq 0}},
\end{aligned}
$$

where $\mathbb{R}_{\geq 0}$ is the set of nonnegative reals. Namely, we have that $\operatorname{VR}(P, r) \subset \operatorname{VR}(P, s)$ and $\operatorname{Alp}(P, r) \subset$ $\operatorname{Alp}(P, s)$ for $r<s$ and $\operatorname{VR}(P, r)=\operatorname{VR}(P, R)$ and $\operatorname{Alp}(P, r)=\operatorname{Alp}(P, R)$ for $r \geq R$, for a sufficiently large $R$ (called saturation time). The radius parameter $r$ is also called time in this paper, following the convention used in persistent homology.

\subsection{General Position}

Let us treat a point cloud $P$ as an ordered set induced by the index $i=1, \ldots, M$. Then, we can assign a single variable $u=\left(u_{1}, \ldots, u_{M}\right) \in \mathbb{R}^{n}$ to $P$, where $n=L M$. Conversely, from a point $u \in \mathbb{R}^{n}$, an ordered subset $P$ in $\mathbb{R}^{L}$ with $|P|=M$ can be constructed. We explicitly denote this correspondence by $u(P)$ and $P(u)$, if necessary, and identify them in the following.

Let $\mathcal{X}=\left(X^{r}\right)_{r \in \mathbb{R}_{\geq 0}}$ be a Vietoris-Rips or an alpha filtration. For each simplex $\sigma \in X^{R}$, we can assign its birth radius $r_{\sigma}$ in the filtration $\mathcal{X}$ by the infimum radius $r$ satisfying $\sigma \in X^{r}$.

In the Vietoris-Rips filtration $\operatorname{VR}(P)$, the birth radius $r_{\sigma}$ of a simplex $\sigma=\left\{u_{i_{0}}, \ldots, u_{i_{\ell}}\right\}$ is a function of $u_{\sigma}=\left(u_{i_{0}}, \ldots, u_{i_{\ell}}\right)$ given by

$$
r_{\sigma}=\frac{1}{2} \max _{0 \leq a<b \leq \ell}\left\|u_{i_{b}}-u_{i_{a}}\right\| .
$$

We call an edge $\left\{u_{i_{a}}, u_{i_{b}}\right\}$ that attains the above maximum an attaching edge of $\sigma$.

Definition 2.1. A configuration $u \in \mathbb{R}^{n}$ is said to be in Vietoris-Rips general position if the following conditions are satisfied:

(i) $u_{i} \neq u_{j}$ for any $i \neq j$,

(ii) $r_{\tau} \neq r_{\tau^{\prime}}$ for any attaching edges $\tau \neq \tau^{\prime}$.

The open set consisting of the points in VietorisRips general position is denoted by $U_{\mathrm{VR}}$.

In the alpha filtration $\operatorname{Alp}(P)$, each simplex $\sigma$ appears as either an attaching simplex or a simplex attached by some attaching simplex $\tau \supset \sigma$. In the latter case, the birth radius $r_{\sigma}$ is given by $r_{\sigma}=r_{\tau}$. 
Definition 2.2. A configuration $u \in \mathbb{R}^{n}$ is said to be in alpha general position if the following conditions are satisfied:

(i) $u$ is in general position in the sense of [11.

(ii) $r_{\tau} \neq r_{\tau^{\prime}}$ for any attaching simplices $\tau \neq \tau^{\prime}$ except 0 -simplices.

The open set consisting of the points in alpha general position is denoted by $U_{\mathrm{Alp}}$.

We note that, in both Vietoris-Rips and alpha filtrations, the condition (ii) implies that an attaching simplex is uniquely determined by its birth radius.

\subsection{Persistent Homology}

We briefly review the definition of persistent homology as a graded module on a monoid ring. Let $\mathcal{X}=\left(X^{r}\right)_{r \in \mathbb{R}_{\geq 0}}$ be a filtration of finite type with a saturation time $R$. For each $X^{r}$, let us denote by $X_{\ell}^{r}$ the set of $\ell$-simplices in $X^{r}$. In the following, we fix an orientation for each simplex $\sigma=\left\{u_{i_{0}}, \ldots, u_{i_{\ell}}\right\}$ by $i_{0}<\cdots<i_{\ell}$, and denote the oriented simplex by $\langle\sigma\rangle=\left\langle u_{i_{0}} \ldots u_{i_{\ell}}\right\rangle$.

Let $k$ be a field, and let us treat $\mathbb{R}_{\geq 0}$ with a monoid structure induced by the addition + . Let $k\left[\mathbb{R}_{>0}\right]$ be a monoid ring. That is, $k\left[\mathbb{R}_{>0}\right]$ is a vector space of formal linear combinations of elements of $\mathbb{R}_{\geq 0}$ equipped with a ring structure

$$
\left(a_{1} r_{1}\right) \cdot\left(a_{2} r_{2}\right)=\left(a_{1} a_{2}\right)\left(r_{1}+r_{2}\right)
$$

for $a_{1}, a_{2} \in k$ and $r_{1}, r_{2} \in \mathbb{R}_{\geq 0}$.

In the following, the elements in $k\left[\mathbb{R}_{\geq 0}\right]$ are expressed by linear combinations of formal monomials $a z^{r}$, where $a \in k, r \in \mathbb{R}_{\geq 0}$, and $z$ is an indeterminate. Then, the product of two elements are defined by linear extension of

$$
a_{1} z^{r_{1}} \cdot a_{2} z^{r_{2}}=a_{1} a_{2} z^{r_{1}+r_{2}} .
$$

Let us denote by $C_{\ell}\left(X^{r}\right)$ the $k$-vector space spanned by the $\ell$-simplices in $X_{\ell}^{r}$. The $\ell$-th chain group $C_{\ell}(\mathcal{X})$ is defined as a graded module on the monoid ring $k\left[\mathbb{R}_{\geq 0}\right]$ by taking a direct sum

$$
C_{\ell}(\mathcal{X})=\bigoplus_{r \in \mathbb{R}_{\geq 0}} C_{\ell}\left(X^{r}\right)=\left\{\left(c_{r}\right) \mid c_{r} \in C_{\ell}\left(X^{r}\right)\right\},
$$

where the action of a monomial $z^{s}$ on $C_{\ell}(\mathcal{X})$ is given by the right shift operator

$$
z^{s} \cdot\left(c_{r}\right)=\left(c_{r}^{\prime}\right) \text { with } c_{r}^{\prime}=\left\{\begin{array}{cc}
c_{r-s}, & r \geq s, \\
0, & r<s .
\end{array}\right.
$$

For a simplex $\sigma$, let us define

$$
\left\langle\langle\sigma\rangle=\left(c_{r}\right), \quad c_{r}=\left\{\begin{array}{cc}
\langle\sigma\rangle, & r=r_{\sigma}, \\
0, & r \neq r_{\sigma} .
\end{array}\right.\right.
$$

We note that $\Xi_{\ell}=\left\{\langle\sigma \sigma\rangle \mid \sigma \in X_{\ell}^{R}\right\}$ forms a basis of $C_{\ell}(\mathcal{X})$. The boundary map $\partial_{\ell}: C_{\ell}(\mathcal{X}) \rightarrow C_{\ell-1}(\mathcal{X})$ is defined by linear extension of

$$
\partial_{\ell}\langle\langle\sigma\rangle\rangle=\sum_{j=0}^{\ell}(-1)^{j} z^{r_{\sigma}-r_{\sigma_{j}}}\left\langle\left\langle\sigma_{j}\right\rangle\right\rangle,
$$

where $\left\langle\sigma_{j}\right\rangle=\left\langle u_{i_{0}} \ldots \widehat{u_{i_{j}}} \ldots u_{i_{\ell}}\right\rangle$ is a face of $\langle\sigma\rangle=$ $\left\langle u_{i_{0}} \ldots u_{i_{\ell}}\right\rangle$, and $\widehat{a}$ means the removal of the vertex $a$.

The cycle group $Z_{\ell}(\mathcal{X})$ and the boundary group $B_{\ell}(\mathcal{X})$ in $C_{\ell}(\mathcal{X})$ are defined by

$$
Z_{\ell}(\mathcal{X})=\operatorname{ker} \partial_{\ell}, \quad B_{\ell}(\mathcal{X})=\operatorname{im} \partial_{\ell+1} .
$$

It follows from $\partial_{\ell} \circ \partial_{\ell+1}=0$ that we have $B_{\ell}(\mathcal{X}) \subset$ $Z_{\ell}(\mathcal{X})$. Then, the $\ell$-th persistent homology is defined by

$$
H_{\ell}(\mathcal{X})=Z_{\ell}(\mathcal{X}) / B_{\ell}(\mathcal{X})
$$

The following theorem is known as the structure theorem of persistent homology.

Theorem 2.3 (4). There uniquely exist indices $s, t \in \mathbb{Z}_{\geq 0}$ and $\left(b_{i}, d_{i}\right) \in \mathbb{R}_{\geq 0}^{2}$, for $i=1, \ldots, s$, with $b_{i}<d_{i}$ and $b_{i} \in \mathbb{R}_{\geq 0}$, for $i=s+1, \ldots, s+t$, such that the following isomorphism holds

$$
H_{\ell}(\mathcal{X}) \simeq \bigoplus_{i=1}^{s}\left(\left(z^{b_{i}}\right) /\left(z^{d_{i}}\right)\right) \oplus \bigoplus_{i=s+1}^{s+t}\left(z^{b_{i}}\right),
$$

where $\left(z^{a}\right)$ expresses an ideal in $k\left[\mathbb{R}_{\geq 0}\right]$ generated by the monomial $z^{a}$. When s or $t$ is zero, the corresponding direct sum is ignored.

The $\ell$-th persistence diagram $D_{\ell}(\mathcal{X})$ of $\mathcal{X}$ is defined as the multiset in $\overline{\mathbb{R}}^{2}$ determined from the decomposition 2.1) by

$$
D_{\ell}(\mathcal{X})=\left\{\left(b_{i}, d_{i}\right) \mid i=1, \ldots, s+t\right\},
$$

where $d_{i}=+\infty$ for $i=s+1, \ldots, s+t$. The pair $\left(b_{i}, d_{i}\right)$ is called a birth-death pair in the $\ell$-th persistence diagram, and $b_{i}, d_{i}$ are called, respectively, the birth and death times of the pair.

\section{Local Properties of the Persistence Map}

\subsection{The Persistence Map}

Let $D_{\ell}\left(\mathcal{X}_{P}\right)$ be the persistence diagram 2.2 of a filtration $\mathcal{X}_{P}$ constructed from a finite set $P$. By 
choosing the birth and death times in 2.2 that are finite, we can express $D_{\ell}\left(\mathcal{X}_{P}\right)$ as a point

$$
v=\left(b_{1}, d_{1}, \ldots, b_{s}, d_{s}, b_{s+1}, \ldots, b_{s+t}\right) \in \mathbb{R}^{m},
$$

where $m=2 s+t$. Then, recalling the identification of $P$ and $u \in \mathbb{R}^{n}$, we can regard the persistent homology as giving a single correspondence

$$
\mathbb{R}^{n} \ni u \longmapsto v \in \mathbb{R}^{m} \text {. }
$$

In this section, we define an appropriate open set $O \subset \mathbb{R}^{n}$ such that this single correspondence is extended to a map $f: O \subset \mathbb{R}^{n} \rightarrow \mathbb{R}^{m}$ computing persistence diagrams with $f(u)=v$.

It should be noted that the dimension $m$ may change for a different choice of $u \in \mathbb{R}^{n}$. For extending the single correspondence to a map into $\mathbb{R}^{m}$, we use a recent result in 13 . Let us first recall some definitions.

For a metric space $\left(X, d_{X}\right)$, the Hausdorff distance $d_{\mathrm{H}}$ between two subsets $A, B \subset X$ is defined by

$$
d_{\mathrm{H}}(A, B)=\max \left\{\sup _{a \in A} d_{X}(a, B), \sup _{b \in B} d_{X}(b, A)\right\},
$$

where $d_{X}(a, B)=\inf _{b \in B} d_{X}(a, b)$ and $d_{X}(b, A)$ is defined symmetrically. The Gromov-Hausdorff distance $d_{\mathrm{GH}}$ between two metric spaces $\left(X, d_{X}\right)$ and $\left(Y, d_{Y}\right)$ is defined by

$$
d_{\mathrm{GH}}(X, Y)=\inf _{f, g} d_{\mathrm{H}}\left(f_{X \rightarrow Z}(X), g_{Y \rightarrow Z}(Y)\right),
$$

where $f_{X \rightarrow Z}$ and $g_{Y \rightarrow Z}$ denote isometric embeddings of $X$ and $Y$ into a metric space $\left(Z, d_{Z}\right)$, respectively, and the Hausdorff distance between $f_{X \rightarrow Z}(X)$ and $g_{Y \rightarrow Z}(Y)$ is measured using the metric $d_{Z}$.

We also recall that the bottleneck distance $d_{\mathrm{b}}$ between two persistence diagrams $D$ and $D^{\prime}$ is defined by

$$
d_{\mathrm{b}}\left(D, D^{\prime}\right)=\inf _{\gamma} \sup _{p \in \widehat{D}}\|p-\gamma(p)\|_{\infty},
$$

where $\widehat{D}$ is a multiset consisting of the points in $D$ and the points on the diagonal $\Delta$ with multiplicity $+\infty$, and $\gamma$ is a bijection between $\widehat{D}$ and $\widehat{D^{\prime}}$. Here, we define the norm $\|p\|_{\infty}=d_{\infty}(p, 0)$ by the distance $d_{\infty}(p, q)=\max \left\{\left|p_{1}-q_{1}\right|,\left|p_{2}-q_{2}\right|\right\}$ on $\overline{\mathbb{R}}^{2}$.

For a multiset $D \subset \overline{\mathbb{R}}^{2}$, let

$$
T_{\epsilon}(D)=\left\{p \in D \mid d_{\infty}(p, \Delta) \geq \epsilon\right\}
$$

be the multiset defined by an $\epsilon$-truncation of $D$ from the diagonal.
Lemma 3.1. Let $D$ be a persistence diagram and $\delta=d_{\infty}(D, \Delta)$. If $d_{\mathrm{b}}\left(D, D^{\prime}\right)<\delta$, then $|D| \leq\left|D^{\prime}\right|$. Furthermore, if $d_{\mathrm{b}}\left(D, D^{\prime}\right)<\epsilon<\delta / 2$, then $|D|=$ $\left|T_{\epsilon}\left(D^{\prime}\right)\right|$.

Proof. Suppose $|D|>\left|D^{\prime}\right|$. Then, there exists a point in $D$ which is mapped to the diagonal $\Delta$ for any bijection $\gamma: \widehat{D} \rightarrow \widehat{D^{\prime}}$. This leads to $\delta \leq d_{\mathrm{b}}\left(D, D^{\prime}\right)$, implying the first statement.

For the proof of the second statement, let $\gamma$ be a bijection $\gamma: \widehat{D} \rightarrow \widehat{D^{\prime}}$ such that

$$
d_{\mathrm{b}}\left(D, D^{\prime}\right) \leq \sup _{p \in \widehat{D}}\|p-\gamma(p)\|_{\infty}<\epsilon .
$$

Suppose $d_{\infty}(\gamma(D), \Delta) \leq \epsilon$. Then, there exists $p \in D$ such that $d_{\infty}(\gamma(p), \Delta) \leq \epsilon$. On the other hand, we have $d_{\infty}(p, \gamma(p)) \leq \sup _{p \in \widehat{D}}\|p-\gamma(p)\|_{\infty}<\epsilon$. This leads to the contradiction

$\delta \leq d_{\infty}(p, \Delta) \leq d_{\infty}(p, \gamma(p))+d_{\infty}(\gamma(p), \Delta)<2 \epsilon<\delta$.

Hence, we have $d_{\infty}(\gamma(D), \Delta)>\epsilon$. Moreover, we have $T_{\epsilon}\left(D^{\prime} \backslash \gamma(D)\right)=\varnothing$, otherwise it gives the contradiction $\sup _{p \in \widehat{D}}\|p-\gamma(p)\|_{\infty} \geq \epsilon$. These two properties show that $|D|=\left|T_{\epsilon}\left(D^{\prime}\right)\right|$.

Now let us apply the result in 13 . Let $P$ and $P^{\prime}$ be two point clouds in $\mathbb{R}^{L}$ with $|P|=\left|P^{\prime}\right|=M$. Then, since $P$ and $P^{\prime}$ are totally bounded, the inequalities

$$
d_{\mathrm{b}}\left(D_{\ell}\left(\mathcal{X}_{P}\right), D_{\ell}\left(\mathcal{X}_{P^{\prime}}\right)\right) \leq d_{\mathrm{GH}}\left(P, P^{\prime}\right)
$$

for Vietoris-Rips filtrations and

$$
d_{\mathrm{b}}\left(D_{\ell}\left(\mathcal{X}_{P}\right), D_{\ell}\left(\mathcal{X}_{P^{\prime}}\right)\right) \leq d_{\mathrm{H}}\left(P, P^{\prime}\right)
$$

for alpha filtrations hold by Theorem 5.2 and Theorem 5.6 in [13, respectively.

Let $\delta=d_{\infty}\left(D_{\ell}\left(\mathcal{X}_{P}\right), \Delta\right)$ and, for $\epsilon<\delta / 2$, let us set

$$
O_{u}^{\epsilon}=\left\{u^{\prime} \in \mathbb{R}^{n} \mid d_{\mathrm{GH}}\left(P(u), P^{\prime}\left(u^{\prime}\right)\right)<\epsilon\right\}
$$

for Vietoris-Rips filtrations, and

$$
O_{u}^{\epsilon}=\left\{u^{\prime} \in \mathbb{R}^{n} \mid d_{\mathrm{H}}\left(P(u), P^{\prime}\left(u^{\prime}\right)\right)<\epsilon\right\}
$$

for alpha filtrations. Then, it follows from Lemma 3.1 that $\left|D_{\ell}\left(\mathcal{X}_{P(u)}\right)\right|=\left|T_{\epsilon}\left(D_{\ell}\left(\mathcal{X}_{P^{\prime}\left(u^{\prime}\right)}\right)\right)\right|$ for any $u^{\prime} \in O_{u}^{\epsilon}$. Hence, given a single persistence correspondence $(u, v)$, we can define a map

$$
\Phi: O_{u}^{\epsilon} \ni u^{\prime} \longmapsto T_{\epsilon}\left(D_{\ell}\left(\mathcal{X}_{P^{\prime}\left(u^{\prime}\right)}\right)\right) \in \mathbb{R}^{m} .
$$

At the end of this section, we show that $O_{u}^{\epsilon}$ is an open set. We first prove the following lemma. 
Lemma 3.2. Let $P=\left\{x_{1}, \ldots, x_{M}\right\}$ and $Q=$ $\left\{y_{1}, \ldots, y_{M}\right\}$ be two point clouds in $\mathbb{R}^{L}$. Set $u=$ $u(P) \in \mathbb{R}^{n}$ and $w=w(Q) \in \mathbb{R}^{n}$. Then,

$$
d_{\mathrm{GH}}(P, Q) \leq d_{\mathrm{H}}(P, Q) \leq d_{\mathbb{R}^{n}}(u, w) .
$$

Proof. By definition of the Gromov-Hausdorff distance, we have

$$
\begin{aligned}
d_{\mathrm{GH}}(P, Q) & \leq d_{\mathrm{H}}(P, Q) \\
& =\max \left\{\max _{i} d_{\mathbb{R}^{L}}\left(x_{i}, Q\right), \max _{i} d_{\mathbb{R}^{L}}\left(P, y_{i}\right)\right\} .
\end{aligned}
$$

Without loss of generality, we assume that $d_{\mathrm{H}}(P, Q)$ is achieved by $x_{i}$. Then,

$$
\begin{aligned}
d_{\mathrm{H}}(P, Q) & =\min _{j} d_{\mathbb{R}^{L}}\left(x_{i}, y_{j}\right) \leq d_{\mathbb{R}^{L}}\left(x_{i}, y_{i}\right) \\
& \leq \sqrt{\sum_{i} d_{\mathbb{R}^{L}}\left(x_{i}, y_{i}\right)^{2}}=d_{\mathbb{R}^{n}}(u, w),
\end{aligned}
$$

and this completes the proof.

Proposition 3.3. $O_{u}^{\epsilon}$ is an open set in $\mathbb{R}^{n}$.

Proof. We consider the case of Rips filtrations. Let us choose $u^{\prime} \in O_{u}^{\epsilon}$ and set $P^{\prime}=P^{\prime}\left(u^{\prime}\right) \subset \mathbb{R}^{L}$. We also take a positive real number $\tau>0$ such that

$$
d_{\mathrm{GH}}\left(P, P^{\prime}\right)+\tau<\epsilon .
$$

We claim that the $\tau$-open neighborhood of $u^{\prime}$ in $\mathbb{R}^{n}$ is contained in $O_{u}^{\epsilon}$. For any $u^{\prime \prime} \in \mathbb{R}^{n}$ with $d_{\mathbb{R}^{n}}\left(u^{\prime}, u^{\prime \prime}\right)<\tau$, we have $d_{\mathrm{GH}}\left(P^{\prime}, P^{\prime \prime}\right) \leq$ $d_{\mathbb{R}^{n}}\left(u^{\prime}, u^{\prime \prime}\right)<\tau$ from Lemma 3.2. Then, it follows from the triangle inequality that

$$
\begin{aligned}
d_{\mathrm{GH}}\left(P, P^{\prime \prime}\right) & \leq d_{\mathrm{GH}}\left(P, P^{\prime}\right)+d_{\mathrm{GH}}\left(P^{\prime}, P^{\prime \prime}\right) \\
& <d_{\mathrm{GH}}\left(P, P^{\prime}\right)+\tau<\epsilon .
\end{aligned}
$$

This proves the claim and, since $u^{\prime}$ is arbitrary, this concludes that $O_{u}^{\epsilon}$ is an open set in $\mathbb{R}^{n}$. We can prove the case for alpha filtrations by replacing $d_{\mathrm{GH}}$ by $d_{\mathrm{H}}$.

\subsection{Decomposition of Persistence Map}

Let $\mathcal{X}=\left(X^{r}\right)_{r \in \mathbb{R}_{\geq 0}}$ be a Vietoris-Rips or alpha filtration with a saturation time $R$, and let us set $W_{\ell}=X_{\ell}^{R}$ and $W=\cup_{\ell} W_{\ell}$. The correspondence (3.1) is decomposed into two parts:

$$
u \stackrel{g}{\longmapsto} r=\left(r_{\sigma}\right)_{\sigma \in W} \stackrel{h}{\longmapsto} v,
$$

where $g$ constructs the simplicial complex filtration, and $h$ computes the persistence diagram. We extend the decomposition $f=h \circ g$ of this single correspondence to that of a map $f$. To this aim, we need to construct a proper subset in $O_{u}^{\epsilon}$ in which the set $W$ is invariant.

In the case of Vietoris-Rips filtration, $W$ is given by all the faces of the $(M-1)$-simplex for $|P|=M$, independently of the configuration $u$. Thus, the decomposition $f=h \circ g$ of the correspondence is extended naturally to the map

$$
O_{\mathrm{VR}} \stackrel{g}{\longrightarrow} \mathbb{R}^{d} \stackrel{h}{\longrightarrow} \mathbb{R}^{m},
$$

where $O_{\mathrm{VR}}=O_{u}^{\epsilon} \cap U_{\mathrm{VR}}, g: O_{\mathrm{VR}} \ni u \longmapsto r=$ $\left(r_{\sigma}\right)_{\sigma \in W} \in \mathbb{R}^{d}$, and $h: \mathbb{R}^{d} \ni r \longmapsto v \in \mathbb{R}^{m}$ with $d=|W|$.

On the other hand, in the case of alpha filtration, note that the set $W$ can generally change depending on the configuration $u$. Recall that the alpha complex $\operatorname{Alp}(P(u), \alpha)$ is a subcomplex of the Delaunay complex $\operatorname{Del}(P(u))$ and $\operatorname{Del}(P(u))=$ $\cup_{\alpha} \operatorname{Alp}(P(u), \alpha)$. Hence, the set $W$ is given by $\operatorname{Del}(P(u))$ in this case.

For a configuration $u$ satisfying the general position assumption, the Delaunay complex $\operatorname{Del}(P(u))$ is stable with respect to small perturbations. Namely, we can construct an open neighborhood $\tilde{O}_{u} \subset \mathbb{R}^{n}$ of $u$ such that the Delaunay complex is invariant in this neighborhood, i.e., $\operatorname{Del}(P(u))=$ $\operatorname{Del}\left(P^{\prime}\left(u^{\prime}\right)\right)$ for all $u^{\prime} \in \tilde{O}_{u}$. Therefore, by setting $O_{\mathrm{Alp}}=O_{u}^{\epsilon} \cap \tilde{O}_{u} \cap U_{\mathrm{Alp}}$, we can extend the decomposition of the map $f$ as

$$
O_{\text {Alp }} \stackrel{g}{\longrightarrow} \mathbb{R}^{d \stackrel{h}{\longrightarrow}} \mathbb{R}^{m} .
$$

In 14, the authors study explicit bounds on the perturbations of $u$ for which the Delaunay complex $\operatorname{Del}(P)$ is invariant.

Remark 3.4. Precisely speaking, $h$ is defined on the image of $g$.

Remark 3.5. When we consider $\ell$-th persistence diagram $D_{\ell}(\mathcal{X})$, it is sufficient to deal only with $W_{\ell-1}$, $W_{\ell}$, and $W_{\ell+1}$.

\subsection{Smoothness of Persistence Map}

\subsubsection{Vietoris-Rips filtration $g$}

Lemma 3.6. On the open set $O_{\mathrm{VR}}$, the map $g$ is of class $C^{\infty}$. 
Proof. For a simplex $\sigma=\left\{u_{i_{0}}, \ldots, u_{i_{\ell}}\right\}$, let $\left\{u_{i_{a}}, u_{i_{b}}\right\}$ be the attaching edge, i.e.,

$$
r_{\sigma}=\frac{1}{2}\left\|u_{i_{b}}-u_{i_{a}}\right\|
$$

From the assumption of the Vietoris-Rips general position, $r_{\sigma}$ is continuously differentiable on $O_{\mathrm{VR}}$ whose entries in the $i_{a}$-th and the $i_{b}$-th coordinates are given by

$$
\frac{\partial r_{\sigma}}{\partial u_{i_{a}}}=\frac{1}{2} \frac{u_{i_{a}}-u_{i_{b}}}{\left\|u_{i_{b}}-u_{i_{a}}\right\|}, \quad \frac{\partial r_{\sigma}}{\partial u_{i_{b}}}=\frac{1}{2} \frac{u_{i_{b}}-u_{i_{a}}}{\left\|u_{i_{b}}-u_{i_{a}}\right\|}
$$

and zero otherwise. It is obvious from the same argument that $r_{\sigma}$ is continuously differentiable arbitrarily many times.

Remark 3.7. It follows from the proof of Lemma 3.6 that breaking the Vietoris-Rips general position assumption immediately makes that map $g$ loose its differentiability.

\subsubsection{Alpha filtration $g$}

Lemma 3.8. On the open set $O_{\mathrm{Alp}}$, the map $g$ is of class $C^{\infty}$.

Proof. Let $\sigma$ be a simplex in the alpha filtration $\operatorname{Alp}(P)$ and $\tau$ be its attaching simplex. Then, it follows from the definition of $\operatorname{Alp}(P)$ that the birth radius $r_{\sigma}$ is given by the radius $\rho_{\tau}$ of the smallest circumsphere of $\tau$.

Let us denote by $u_{i}=\left(u_{i, 1}, u_{i, 2}, u_{i, 3}\right) \in \mathbb{R}^{3}$ the coordinate of each point $u_{i}$. Let $u_{i, 0}$ and $u_{i, 4}$ be given by $u_{i, 0}=1$ and $u_{i, 4}=\sum_{j=1}^{3} u_{i, j}^{2}$, respectively. We define the determinant

$$
M_{j_{1} j_{2} \ldots j_{k}}^{i_{1} i_{2} \ldots i_{k}}=\operatorname{det}\left[\begin{array}{cccc}
u_{i_{1}, j_{1}} & u_{i_{1}, j_{2}} & \cdots & u_{i_{1}, j_{k}} \\
u_{i_{2}, j_{1}} & u_{i_{2}, j_{2}} & \cdots & u_{i_{2}, j_{k}} \\
\vdots & \vdots & \ddots & \vdots \\
u_{i_{k}, j_{1}} & u_{i_{k}, j_{2}} & \cdots & u_{i_{k}, j_{k}}
\end{array}\right] .
$$

Then, the formulas for $\rho_{\tau}$ for $|\tau|=2,3,4$ are given in [11] as

$$
\begin{aligned}
\tau & =\left\{u_{i}, u_{j}\right\}: \\
\rho_{\tau}^{2} & =\frac{\left(M_{10}^{i j}\right)^{2}+\left(M_{20}^{i j}\right)^{2}+\left(M_{30}^{i j}\right)^{2}}{4}, \\
\tau & =\left\{u_{i}, u_{j}, u_{k}\right\}: \\
\rho_{\tau}^{2} & =\frac{\left(\sum_{z=1}^{3}\left(M_{z 0}^{i j}\right)^{2}\right) \cdot\left(\sum_{z=1}^{3}\left(M_{z 0}^{j k}\right)^{2}\right) \cdot\left(\sum_{z=1}^{3}\left(M_{z 0}^{k i}\right)^{2}\right)}{4\left(\left(M_{230}^{i j k}\right)^{2}+\left(M_{130}^{i j k}\right)^{2}+\left(M_{120}^{i j k}\right)^{2}\right)}, \\
\tau & =\left\{u_{i}, u_{j}, u_{k}, u_{\ell}\right\}: \\
\rho_{\tau}^{2} & =\frac{\left(M_{2340}^{i j k \ell}\right)^{2}+\left(M_{1340}^{i j k \ell}\right)^{2}+\left(M_{1240}^{i j k \ell}\right)^{2}+4 \cdot M_{1230}^{i j k \ell} \cdot M_{1234}^{i j k \ell}}{4 \cdot\left(M_{1230}^{i j k \ell}\right)^{2}} .
\end{aligned}
$$

It follows from the definition of the alpha general position and the above formula that $g$ is of class $C^{\infty}$ on $O_{\mathrm{Alp}}$.

Remark 3.9. It follows from the proof of Lemma 3.8 that breaking the alpha general position assumption immediately makes that map $g$ loose its differentiability.

\subsubsection{The map $h$}

Let us next study the map $h$ and its differentiability. The map $h$ can be computed by Algorithm 1 below, which consists of three parts and is based on [15] and [4. Each procedure is presented in what follows.

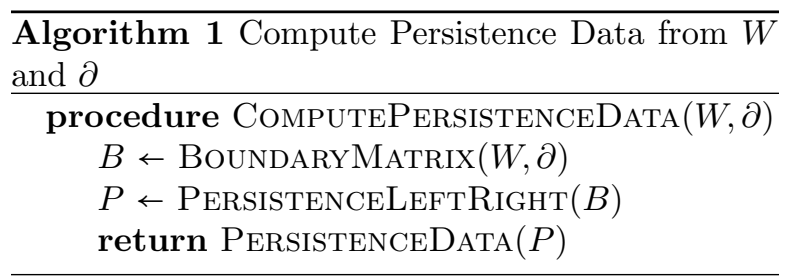

BoundaryMatrix. For a matrix $A=\left(A_{i j}\right)$, let us denote its $j$-th column of $A$ by $A_{j}$, and for a nonzero column $A_{j}$, set $\operatorname{pivot}\left(A_{j}\right):=\max \left\{i \mid A_{i j} \neq 0\right\}$, called the pivot index.

Let us order the set $W$ of all simplices, $\sigma_{1}<$ $\sigma_{2}<\cdots<\sigma_{K}$, by the lexicographical order of $\left(r_{\sigma}, \operatorname{dim} \sigma\right) \in \mathbb{R}_{\geq 0} \times \mathbb{Z}$. If two (or more) simplices $\sigma, \sigma^{\prime}$ appear at the same birth radius with the same dimension, we order them by an appropriate rule.

In this order, a subset $\left\{\sigma_{1}, \cdots, \sigma_{k}\right\}$ for any $k$ is a subcomplex of $W$ in both the Vietoris-Rips and the alpha filtrations.

Let $C$ be the vector space spanned by $\left\{\sigma_{1}, \ldots, \sigma_{K}\right\}$. A matrix representation $B=\left(B_{i j}\right)$ of the boundary map $\partial: C \rightarrow C$ is constructed in such a way that for an $\ell$-simplex $\sigma_{i}=\left\{u_{i_{0}}, \ldots, u_{i_{\ell}}\right\}$ with $i_{0}<\cdots<i_{\ell}$, the $(i, j)$-entry is given by

$$
B_{i j}= \begin{cases}(-1)^{k}, & \sigma_{j}=\left\{u_{i_{0}}, \ldots, \widehat{u_{i_{k}}}, \ldots, u_{i_{\ell}}\right\}, \\ 0, & \text { otherwise. }\end{cases}
$$

PersistenceLeftRight. A column operation of the form $A_{j} \leftarrow A_{j}+\lambda A_{k}$ is called a left-to-right operation if $k<j$. We call a matrix $A^{\prime}$ derived from $A$ if $A^{\prime}$ can be transformed from $A$ by left-toright operations. We call a matrix $A^{\prime}$ reduced if no two non-zero columns have the same pivot index. 
If a reduced matrix $A^{\prime}$ is derived from $A$, we call it a reduction of $A$. In this case, we define

$P_{A^{\prime}}=\left\{(i, j) \mid A_{j}^{\prime} \neq 0\right.$ and $\left.i=\operatorname{pivot}\left(A_{j}^{\prime}\right)\right\}$,

$E_{A^{\prime}}=\left\{i \mid 1 \leq i \leq K, i \neq i^{\prime}\right.$ and $i \neq j^{\prime}$ for $\left.\forall\left(i^{\prime}, j^{\prime}\right) \in P_{A^{\prime}}\right\}$

For a reduction $B^{\prime}$ of the matrix representation $B$, it follows from [4] that we can find a basis $\left\{w_{1}, \ldots, w_{K}\right\}$ of $C$ satisfying: (i) the subspace spanned by $\left\{\sigma_{1}, \ldots, \sigma_{k}\right\}$ is equal to the subspace spanned by $\left\{w_{1}, \ldots, w_{k}\right\}$ for any $k$, and (ii) $\partial w_{j}$ is given by

$$
\partial w_{j}=\left\{\begin{array}{ll}
w_{i}, & (i, j) \in P_{B^{\prime}} \\
0, & \text { otherwise }
\end{array} .\right.
$$

Algorithm 2 (a modification of Algorithm 1 in [15]) shows a simple algorithm to compute $P_{B^{\prime}}$ of the matrix $B$. Note that $E_{B^{\prime}}$ is easily computable from $P_{B^{\prime}}$. The algorithm processes columns from left to right; for each column, other columns are added from the left until a new pivot index appears or the column becomes zero.

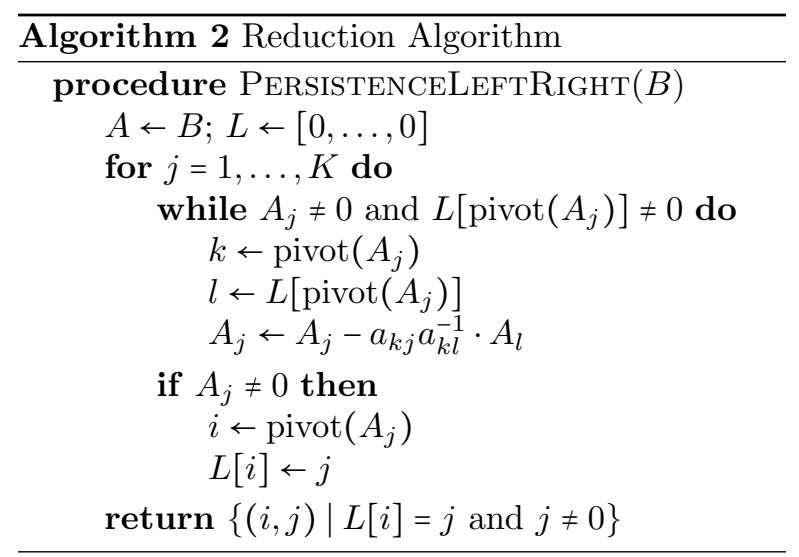

PersistenceData. The basis $\left\{w_{1}, \ldots, w_{K}\right\}$ represents the decomposition of the persistent homology, and hence, we obtain the persistence data from $P_{B^{\prime}}$ as follows:

$$
\begin{cases}\left(r_{\sigma_{i}}, r_{\sigma_{j}}\right), & \text { for }(i, j) \in P_{B^{\prime}} \text { and } \operatorname{dim} \sigma_{i}=\ell, \\ \left(r_{\sigma_{i}}, \infty\right), & \text { for } i \in E_{B^{\prime}} \text { and } \operatorname{dim} \sigma_{i}=\ell\end{cases}
$$

Note that the persistence data is independent of the choice of algorithm from the unique decomposition of persistent homology.

As a result of the above algorithms, the map $h$ : $\mathbb{R}^{d} \rightarrow \mathbb{R}^{m}$ is expressed by

$$
h(r)=\left[\begin{array}{c}
h_{\mathrm{fin}}(r) \\
h_{\mathrm{inf}}(r)
\end{array}\right]
$$

with

$$
h_{\mathrm{fin}}(r)=\left[\begin{array}{c}
r_{\sigma_{i_{1}}} \\
r_{\sigma_{j_{1}}} \\
\vdots \\
r_{\sigma_{i_{s}}} \\
r_{\sigma_{j_{s}}}
\end{array}\right], \quad h_{\mathrm{inf}}(r)=\left[\begin{array}{c}
r_{\sigma_{i_{s+1}}} \\
\vdots \\
r_{\sigma_{i_{s+t}}}
\end{array}\right]
$$

where $\left\{\left(i_{1}, j_{1}\right), \ldots,\left(i_{s}, j_{s}\right)\right\}=\left\{(i, j) \in P_{R} \mid \operatorname{dim} \sigma_{i}=\right.$ $\ell$ and $\left.\left(r_{\sigma_{i}}, r_{\sigma_{j}}\right) \in U_{\epsilon}\right\}, U_{\epsilon}=\left\{p \in \overline{\mathbb{R}}^{2} \mid d_{\infty}(p, \Delta) \geq \epsilon\right\}$, and $\left\{i_{s+1}, \cdots, i_{s+t}\right\}=\left\{i \in E_{R} \mid \operatorname{dim} \sigma_{i}=\ell\right\}$. Hence, we have $m=2 s+t$. The condition $\left(r_{\sigma_{i}}, r_{\sigma_{j}}\right) \in U_{\epsilon}$ guarantees the uniqueness of $m$ from Lemma 3.1 .

The following lemma is derived easily from the explicit form of $h(r)$.

Lemma 3.10. The map $h$ is of class $C^{\infty}$ on $\mathbb{R}^{d}$.

We remark that it is sufficient to treat the $\ell-1$, $\ell$, and the $(\ell+1)$-simplices in Algorithm 2, if we want the persistence diagram $D_{\ell}$ for a single value of $\ell$.

\subsubsection{The map $f$}

It follows from the chain rule $D f(u)=D h(r) \circ$ $D g(u)$ that the explicit form of the derivative $D f(u)$ is given by

$$
D f(u)=\left[\begin{array}{c}
D f_{\text {fin }}(u) \\
D f_{\text {inf }}(u),
\end{array}\right]
$$

with

$$
D f_{\text {fin }}(u)=\left[\begin{array}{c}
\frac{\partial r_{\sigma_{i_{1}}}}{\partial u} \\
\frac{\partial r_{\sigma_{1}}}{\partial u} \\
\vdots \\
\frac{\partial r_{\sigma_{i_{s}}}}{\partial u} \\
\frac{\partial r_{\sigma_{s}}}{\partial u}
\end{array}\right], \quad D f_{\mathrm{inf}}(u)=\left[\begin{array}{c}
\frac{\partial r_{\sigma_{i_{s+1}}}}{\partial u} \\
\vdots \\
\frac{\partial r_{\sigma_{s+t}}}{\partial u}
\end{array}\right] .
$$

Proposition 3.11. The map $f$ is of class $C^{\infty}$ on $O_{\mathrm{VR}}$ and $O_{\mathrm{Alp}}$. Moreover, the derivatives are independent of the choice of algorithms up to permutations of coordinates in $\mathbb{R}^{m}$.

Proof. The first statement follows from the chain rule and Lemmas 3.6, 3.8, and 3.10. For the second statement, let us assume two different expressions $h$ and $h^{\prime}$. From the uniqueness of the persistence data, we can express $h$ and $h^{\prime}$ by appropriate per- 
mutations if necessary as

$$
\begin{aligned}
h(u)= & {\left[\begin{array}{c}
h_{\mathrm{fin}}(u) \\
h_{\mathrm{inf}}(u)
\end{array}\right], } \\
h_{\mathrm{fin}}(u) & =\left[\begin{array}{c}
r_{\sigma_{i_{1}}} \\
r_{\sigma_{j_{1}}} \\
\vdots \\
r_{\sigma_{i_{s}}} \\
r_{\sigma_{j_{s}}}
\end{array}\right], h_{\mathrm{inf}}(u)=\left[\begin{array}{c}
r_{\sigma_{i_{s+1}}} \\
\vdots \\
r_{\sigma_{i_{s+t}}}
\end{array}\right],
\end{aligned}
$$

and

$$
\begin{aligned}
& h^{\prime}(u)= {\left[\begin{array}{c}
h_{\mathrm{fin}}^{\prime}(u) \\
h_{\mathrm{inf}}^{\prime}(u)
\end{array}\right], } \\
& h_{\mathrm{fin}}^{\prime}(u)=\left[\begin{array}{c}
r_{\sigma_{i_{1}^{\prime}}} \\
r_{\sigma_{j_{1}^{\prime}}} \\
\vdots \\
r_{\sigma_{i_{s}^{\prime}}} \\
r_{\sigma_{j_{s}^{\prime}}}
\end{array}\right], h_{\mathrm{inf}}^{\prime}(u)=\left[\begin{array}{c}
r_{\sigma_{i_{s+1}^{\prime}}} \\
\vdots \\
r_{\sigma_{i_{s+t}^{\prime}}}
\end{array}\right]
\end{aligned}
$$

with

$$
r_{\sigma_{i_{k}}}=r_{\sigma_{i_{k}^{\prime}}}, \quad r_{\sigma_{j_{k}}}=r_{\sigma_{j_{k}^{\prime}}}
$$

for all $k$. On the other hand, it follows from the definitions of the Vietoris-Rips and the alpha general positions of $u$ that there uniquely exist the attaching simplices $\eta_{k}$ and $\xi_{k}$ such that

$$
r_{\eta_{k}}=r_{\sigma_{i_{k}}}=r_{\sigma_{i_{k}^{\prime}}}, \quad r_{\xi_{k}}=r_{\sigma_{j_{k}}}=r_{\sigma_{j_{k}^{\prime}}}
$$

for each $k$. Hence, this leads to

$$
\frac{\partial r_{\eta_{k}}}{\partial u}=\frac{\partial r_{\sigma_{i_{k}}}}{\partial u}=\frac{\partial r_{\sigma_{i_{k}^{\prime}}}}{\partial u}, \quad \frac{\partial r_{\xi_{k}}}{\partial u}=\frac{\partial r_{\sigma_{j_{k}}}}{\partial u}=\frac{\partial r_{\sigma_{j_{k}^{\prime}}}}{\partial u},
$$

and completes the proof of the second statement.

Remark 3.12. Since $(3.2)$ and $(3.3)$ are 1-Lipschitz, it is reasonable to have differentiability from Rademacher's theorem 16. The discussion in this section provides a constructive proof of this fact, and furthermore, shows that the derivatives are independent of the choice of algorithm.

\section{Continuation}

\subsection{Continuation by Newton-Raphson Method}

We first recall the standard Newton-Raphson continuation method [10]. Let $U$ be an open set in $\mathbb{R}^{n}$ and $\varphi: U \times \mathbb{R} \rightarrow \mathbb{R}^{n}$ be a $C^{1}$ mapping. Suppose that $(\bar{u}, \bar{\lambda}) \in U \times \mathbb{R}$ satisfies $\varphi(\bar{u}, \bar{\lambda})=0$. Our purpose is to solve $\varphi(u, \lambda)=0$ with respect to $u \in U$ for a given $\lambda$. The existence and the local uniqueness of the solution $u=u_{\lambda}$ is guaranteed by the implicit function theorem when $D_{u} \varphi(\bar{u}, \bar{\lambda})$ is regular and $\lambda$ is sufficiently close to $\bar{\lambda}$.

In practical computations, we find the solution $u_{\lambda}$ for each $\lambda$ by iteratively solving linear equations as follows. By taking an appropriate initial point $u^{(0)}$, the linear approximation of $\varphi(u, \lambda)$ at each iteration step $j$ is given by

$$
\varphi(u, \lambda) \approx \varphi\left(u^{(j)}, \lambda\right)+D_{u} \varphi\left(u^{(j)}, \lambda\right)\left(u-u^{(j)}\right) .
$$

Setting the right hand side to be zero

$$
\varphi\left(u^{(j)}, \lambda\right)+D_{u} \varphi\left(u^{(j)}, \lambda\right)\left(u-u^{(j)}\right)=0,
$$

we obtain an update of the approximate solution

$$
u^{(j+1)}=u^{(j)}-D_{u} \varphi\left(u^{(j)}, \lambda\right)^{-1} \varphi\left(u^{(j)}, \lambda\right) .
$$

This iteration method is called the NewtonRaphson Method, and the convergence of the iterations under suitable regularity of derivatives is well studied (e.g., [17]).

The continuation of the solution $(\bar{u}, \bar{\lambda})$ to a parameter $\lambda^{\prime}$ is achieved by gradually changing the parameter $\lambda$. That is, for $\lambda_{0}=\bar{\lambda}<\lambda_{1}<\cdots<\lambda_{N}=\lambda^{\prime}$, the Newton-Raphson method is applied for each parameter $\lambda_{i}$ by setting $u^{(0)}=u_{\lambda_{i-1}}$ with $u_{\lambda_{0}}=\bar{u}$

\subsection{Newton-Raphson Method by Pseudo-Inverse}

Let $f: U \rightarrow \mathbb{R}^{m}$ be a persistence map defined on an open set $U \subset \mathbb{R}^{n}$. Let us define a map

$$
F: U \times \mathbb{R}^{m} \rightarrow \mathbb{R}^{m}
$$

by $F(u, v)=f(u)-v$. For a given pair $(\bar{u}, \bar{v})$ satisfying $F(\bar{u}, \bar{v})=0$ and $v$ close to $\bar{v}$, our interest is to solve $F(u, v)=0$ with respect to $u \in U$. The existence of the solution is guaranteed when $D f(\bar{u})$ is surjective and $v$ is sufficiently close to $\bar{v}$. Hence, for the rest of the paper, we add the assumption that $m \leq n$. In this case, the solutions form an $n-m$ dimensional manifold.

The basic strategy to solve $F(u, v)=0$ is the same as in Section 4.1, and we derive an iteration method for $u^{(j)}, j=0,1, \ldots, N$, with $u^{(0)}=\bar{u}$ converging to the solution. Namely, the linear approximation of $F$ at $u^{(j)}$ leads to

$$
D f\left(u^{(j)}\right)\left(u-u^{(j)}\right)+F\left(u^{(j)}, v\right)=0,
$$

where $D f\left(u^{(j)}\right)=D_{u} F\left(u^{(j)}, v\right)$, and we derive an update $u^{(j+1)}$ by solving the linear equation with 
respect to $u$. However, we note that the linear equation (4.3) is defined having domain and image with different dimensions in general. Thus $D f\left(u^{(j)}\right)$ is an $m \times n$ rectangular matrix with $m \leq n$.

In general, let us consider a linear equation

$$
A x=b, \quad A \in M_{m, n}(\mathbb{R}), b \in \mathbb{R}^{m}
$$

with $m \leq n$. For solving this type of linear equations, we first recall the concept of pseudo-inverse and explain its relation to solutions of the linear system 4.4.

For a matrix $A \in M_{m, n}(\mathbb{R})$, there exists a unique matrix $X \in M_{n, m}(\mathbb{R})$ satisfying the so-called Penrose equations:

$$
\begin{aligned}
& A X A=A, \quad X A X=X, \\
& (A X)^{T}=A X, \quad(X A)^{T}=X A,
\end{aligned}
$$

where $A^{T}$ is the transpose matrix of $A$. The unique matrix solution $X$ is called the pseudo-inverse of $A$ and denoted by $A^{\dagger}$.

An explicit formula to construct $A^{\dagger}$ is given for instance in 18, 19. Assume that the matrix $A \in M_{m, n}(\mathbb{R})$ has the rank $k \leq m$. Then, it has a singular value decomposition (SVD) of the form $A=V \Sigma W^{T}$, where $V$ and $W$ are orthogonal matrices, and the matrix $\Sigma=\left(\sigma_{i, j}\right) \in M_{m, n}(\mathbb{R})$ has $\sigma_{i, j}=0$ for all $i \neq j$, and $\sigma_{1,1} \geq \sigma_{2,2} \geq \cdots \geq \sigma_{k, k}>$ $\sigma_{k+1, k+1}=\cdots=\sigma_{m, m}=0$. The numbers $\sigma_{i}:=\sigma_{i, i}$, $i=1, \ldots, m$, are called the singular values of the matrix $A$. From the SVD of the matrix $A$, the pseudo-inverse $A^{\dagger}$ can be obtained by the formula

$$
A^{\dagger}=W \Sigma^{\dagger} V^{T},
$$

where $\Sigma^{\dagger}=\left(\sigma_{i, j}^{\dagger}\right)$ is the $n \times m$ matrix with $\sigma_{i, j}^{\dagger}=0$ for all $i \neq j, \sigma_{i, i}^{\dagger}=\sigma_{i}^{-1}$ for $i=1, \ldots, k$, and $\sigma_{i, i}^{\dagger}=0$ for $k<i \leq m$.

Equation 4.4 has a solution for $x$ if and only if $b \in \operatorname{im}(A)$. In such a case, there is a unique minimum norm solution $x$ of 4.4 , meaning that the Euclidean norm $\|x\|$ is minimum among all the solutions of 4.4). If $b \notin \operatorname{im}(A)$, then a least-squares solution $x$ of (4.4) is a vector minimizing the error $\|A x-b\|$. The following proposition provides us with relations between the pseudo-inverse and solutions of the linear equation. For a proof, the reader may refer to [18] for instance.

Proposition 4.1. Let $A \in M_{m, n}(\mathbb{R})$ and $b \in \mathbb{R}^{m}$. If $b \in \operatorname{im}(A)$, then the unique minimum norm solution of $A x=b$ is given by $x=A^{\dagger} b$. If $b \notin \operatorname{im}(A)$, among the least-squares solutions of $A x=b, A^{\dagger} b$ is the one of minimum norm.

This proposition provides us with a method for finding the minimum norm solution of the equation 4.3. . Namely, we update the approximate solution $u^{(3)}$ by

$$
u^{(j+1)}=u^{(j)}-A_{j}^{\dagger} F\left(u^{(j)}, v\right),
$$

where $A_{j}=D f\left(u^{(j)}\right)$. Note that, from the minimality condition on the norm, the update $u^{(j+1)}$ is chosen to be closest to $u^{(j)}$, and this is a natural choice for the purpose of continuation.

The convergence of the iterations 4.5 is studied in 20] (see also 21]), and we summarize it as follows.

Proposition 4.2. Let $r>0$ be a positive real number such that $f \in C^{1}\left(B_{r}(\bar{u})\right)$. Let $\alpha, \beta$ be positive constants such that, for all $u, w \in B_{r}(\bar{u})$ with $u-w \in \operatorname{im} D f(w)^{T}$, the followings hold:

$$
\begin{aligned}
& \|D f(w)(u-w)-f(u)+f(w)\| \leq \alpha\|u-w\|, \\
& \left\|\left(D f(w)^{\dagger}-D f(u)^{\dagger}\right) f(u)\right\| \leq \beta\|u-w\|, \\
& \alpha\left\|D f(z)^{\dagger}\right\|+\beta=\gamma<1 \quad \text { for all } z \in B_{r}(\bar{u}), \\
& \left\|D f(\bar{u})^{\dagger}\right\| \cdot\|f(\bar{u})\|<(1-\gamma) r .
\end{aligned}
$$

Then the iteration 4.5 converges to a solution of

$$
D f(u)^{T} F(u, v)=0
$$

which lies in $B_{r}(\bar{u})$.

When $m=n$, this proposition provides a criterion for the convergence of the Newton-Raphson method 4.1.

For $\operatorname{rank}(D f(u))=m$, the iteration converges to a solution of $F(u, v)=0$. On the other hand, when $\operatorname{rank}(D f(u))<m$, the convergent point $u$ does not necessarily satisfy $F(u, v)=0$, but only implies $F(u, v) \in \operatorname{ker} D f(u)$. Thus, in our continuation method, we suppose that all the singular values $\sigma_{1}, \ldots, \sigma_{m}$ are positive.

\subsection{Continuation of Point Clouds}

We use the iteration 4.5 for continuations of a point cloud. Let $f: U \rightarrow \mathbb{R}^{m}$ be a persistence map. Suppose that $\left(u_{s}, v_{s}\right) \in U \times \mathbb{R}^{m}$ is a pair satisfying $f\left(u_{s}\right)=v_{s}$. This pair can be regarded as the initial point of the continuation. Our task is to continuate it to a target persistence data $v_{t} \in \mathbb{R}^{m}$ and obtain $u_{t} \in \mathbb{R}^{n}$ satisfying $f\left(u_{t}\right)=v_{t}$. 
As the simplest way of the continuation, we divide the line between $v_{s}$ and $v_{t}$ equally into $N$ small segments, and for each $v=v_{s}+k \Delta v, k=1, \ldots, N$, where $\Delta v=\frac{v_{t}-v_{s}}{N}$, we apply the iteration method 4.5 and obtain the point cloud $u$ satisfying $f(u)=$ $v$. This process is summarized in Algorithm 3 .

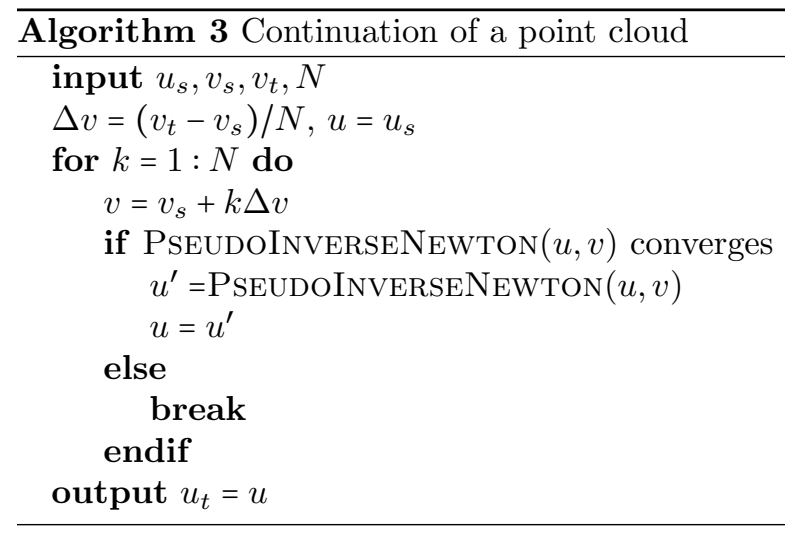

We can, of course, adaptively choose the length of $\Delta v$ at each continuation step. Furthermore, we can also adopt an appropriate curve connecting $v_{s}$ to $v_{t}$ if necessary.

We also note that the image of $f$ is not generally equal to the target space $\mathbb{R}^{m}$. Hence, if we choose $v_{t}$ in $\mathbb{R}^{m} \backslash \operatorname{im}(f)$, the continuation fails at some $v=v_{s}+k \Delta v$. See Section 5.2 for such an example.

It is often the case in practical problems that the point clouds need to satisfy some constraints $g_{i}(u)=0, i=1, \ldots, r$ (e.g., conservation laws in mechanics). In such a case, we need to solve the continuation under these constraints, and its modification is straightforward. Namely, we first extend the original setting 4.2 to

$$
\tilde{F}: U \times \mathbb{R}^{m} \rightarrow \mathbb{R}^{m+r}
$$

by $\tilde{F}(u, v)=\left(f(u)-v, g_{1}(u), \ldots, g_{r}(u)\right)$. Then, by replacing $A_{j}$ and $F$ in 4.5 with $\tilde{A}_{j}=D_{u} \tilde{F}$ and $\tilde{F}$, respectively, we obtain the appropriate formulas for continuation of point clouds under constraints $g_{i}(u)=0, i=1, \ldots, r$.

\subsection{Symmetry}

It should be noted that we need to remove symmetries induced by translations and rotations in order to isolate a solution $u$ of $f(u)=v$. For a point cloud $P$ in $\mathbb{R}^{3}$, the following restrictions will remove these symmetries:

(i) Fix one of the vertices in $P$ at the origin of $\mathbb{R}^{3}$, say $u_{0} \equiv(0,0,0)$. (ii) Select one of the vertices in $P \backslash\left\{u_{0}\right\}$, say $u_{1}$. This vertex is supposed to move only on the line defined by $\overrightarrow{u_{0} u_{1}}$ during the continuation.

(iii) Select one of the vertices in $P \backslash\left\{u_{0}, u_{1}\right\}$, say $u_{2}$. This vertex is supposed to move only on the plane defined by $\overrightarrow{u_{0} u_{1}}, \overrightarrow{u_{0} u_{2}}$ during the continuation.

The first restriction eliminates translation symmetries and the second and third restriction eliminate rotation symmetries.

In practice we choose our basis of the coordinate system $(x, y, z) \in \mathbb{R}^{3}$ in such a way that, in addition to $u_{0}$ being fixed at the origin, we have that $u_{1}$ stays on the $x$-axis and $u_{2}$ stays on the $(x, y)$-plane during the continuation. Hence, in the following, let us redefine $n=3 M-6$ and

$$
u=\left(u_{1}, u_{2}, u_{3}, \ldots, u_{M}\right),
$$

where $u_{1}=x_{1}, u_{2}=\left(x_{2}, y_{2}\right), u_{i}=\left(x_{i}, y_{i}, z_{i}\right)$ for $3 \leq$ $i \leq M$.

For a general point cloud in $\mathbb{R}^{L}$, these restriction to eliminate the symmetries should be appropriately modified.

\subsection{Non-convergence and Zero Singular Values}

The Newton-Raphson method by pseudo-inverse does not work well when the matrix $A_{j}$ in 4.5 has a singular value close to zero. In this section, we discuss those cases in the alpha and Vietoris-Rips filtrations.

\subsubsection{For the case of alpha filtrations}

For the case of alpha filtrations, we show that a singular value close to zero appears when there is a birth-death pair $(b, d)$ with $b \approx d$ in the persistence diagram $D_{\ell}(\operatorname{Alp}(P))$. Here, we impose the natural assumptions that a point cloud $P$ in $\mathbb{R}^{L}(L=2,3)$ satisfies the general position assumption and consists of at least $L+1$ points.

Let $\operatorname{Alp}(P, \alpha+)$ be the alpha complex constructed by simplices whose birth radii are smaller than or equal to $\alpha$. Notice that this is different from $\operatorname{Alp}(P, \alpha)$, where $\alpha$ is equal to the birth radius of a simplex.

Proposition 4.3. Let $P$ be a point cloud and let $(b, d)$ be a birth-death pair in $D_{\ell}(\operatorname{Alp}(P))(\ell=1,2)$. If the birth radius of any simplex is not contained in the open interval $(b, d)$, there exist an attaching $\ell$-simplex $\sigma$ and an attaching $(\ell+1)$-simplex $\tau$ such that $r_{\sigma}=b, r_{\tau}=d$, and $\tau \supset \sigma$. 
To prove the proposition, we need the following lemma.

Lemma 4.4. Let $\sigma$ be a simplex and $\tau$ be its attaching simplex with $\sigma \neq \tau$. Then, the inclusion from $\left|\operatorname{Alp}\left(P, r_{\tau}\right)\right|$ to $\left|\operatorname{Alp}\left(P, r_{\tau}+\right)\right|$ is a deformation retract. In particular, $r_{\tau}$ is neither a birth time nor a death time.

To prove the lemma, we recall some properties about Voronoi decomposition 22. For $k+1$ points $\left\{u_{0}, \ldots, u_{k}\right\}$ in $P$, let $V\left(u_{0}, \cdots, u_{k}\right)$ be defined as

$$
\begin{aligned}
& V\left(u_{0}, \cdots, u_{k}\right) \\
= & \left\{x \in \mathbb{R}^{L} \mid d\left(x, u_{0}\right)=\cdots=d\left(x, u_{k}\right),\right. \text { and } \\
& \left.d\left(x, u_{0}\right) \leq d(x, u) \text { for } \forall u \in P \backslash\left\{u_{0}, \ldots, u_{k}\right\}\right\} .
\end{aligned}
$$

For $k=0$, the set $V\left(u_{0}\right)$ is called a Voronoi cell. From the theory of Voronoi decompositions and Delaunay triangulations, we have the following facts:

1. $V\left(u_{0}, \cdots, u_{k}\right)=V\left(u_{0}\right) \cap \cdots \cap V\left(u_{k}\right)$.

2. $\left\{u_{0}, \cdots, u_{k}\right\}$ forms a Delaunay $k$-simplex if and only if $V\left(u_{0}, \cdots, u_{k}\right)$ is not empty.

3. $V\left(u_{0}, \cdots, u_{k}\right)$ is closed, convex, and contained in a $(L-k)$-dimensional affine subspace $W\left(u_{0}, \cdots, u_{k}\right)=\left\{x \in \mathbb{R}^{L} \mid d\left(x, u_{0}\right)=\right.$ $\left.\cdots=d\left(x, u_{k}\right)\right\} . W\left(u_{0}, \cdots, u_{k}\right)$ is orthogonal to the $k$-dimensional affine subspace spanned by $\left\{u_{0}, \ldots, u_{k}\right\}$.

4. The boundary of $V\left(u_{0}, \cdots, u_{k}\right)$ relative to $W\left(u_{0}, \cdots, u_{k}\right)$ is $\bigcup_{v \in Y\left(u_{0}, \cdots, u_{k}\right)} V\left(u_{0}, \cdots, u_{k}, v\right)$, where $Y\left(u_{0}, \cdots, u_{k}\right)=\left\{v \in P \backslash\left\{u_{0}, \cdots, u_{k}\right\} \mid\right.$ $\left.V\left(u_{0}, \cdots, u_{k}\right) \cap V(v) \neq \varnothing\right\}$.

5. If $V\left(u_{0}, \cdots, u_{k}\right)$ is not empty, $Y\left(u_{0}, \cdots, u_{k}\right)$ is not empty.

6. A Delaunay $k$-simplex $\left\{u_{0}, \ldots, u_{k}\right\}$ is attaching if and only if this simplex and $V\left(u_{0}, \cdots, u_{k}\right)$ have a non-empty intersection.

Proof. (Lemma 4.4) First we consider the case of $L=2, \operatorname{dim} \sigma=1$, and $\operatorname{dim} \tau=2$. Let $u_{1}, u_{2}$ be the endpoints of $\sigma$ and $u_{0}$ be the other vertex of $\tau$. From the facts 2 and 3 above, $V\left(u_{1}, u_{2}\right)$ is not empty and contained in the perpendicular bisector of $\sigma$. From the fact 5, $Y\left(u_{1}, u_{2}\right)$ is not empty and $V\left(u_{1}, u_{2}\right)$ is a line with one endpoint or with two endpoints. Since $\sigma$ is not attaching, $V\left(u_{1}, u_{2}\right) \cap \sigma=\varnothing$ from the fact 6 . Let $E$ be the endpoint of $V\left(u_{1}, u_{2}\right)$ close to $\sigma$, which is given by
$E=V\left(u_{1}, u_{2}, u_{0}\right)$ from the fact 4. Then, from the definition of $E=V\left(u_{1}, u_{2}, u_{0}\right)$ and the general position assumption, the following holds:

$$
\begin{aligned}
& d\left(E, u_{1}\right)=d\left(E, u_{2}\right)=d\left(E, u_{0}\right), \\
& d(E, u)>d\left(E, u_{1}\right) \text { for } \forall u \in P \backslash\left\{u_{0}, u_{1}, u_{2}\right\} .
\end{aligned}
$$

We take a new orthogonal coordinate system on $\mathbb{R}^{2}$ satisfying

- $E=(0,0)$

- $V\left(u_{1}, u_{2}\right)$ is contained in $\{(t, 0) \mid t \leq 0\}$.

In this coordinate, $u_{0}, u_{1}$, and $u_{2}$, are described as

$$
\begin{aligned}
& u_{0}=\left(r \cos \theta_{0}, r \sin \theta_{0}\right), \\
& u_{1}=\left(r \cos \theta_{1}, r \sin \theta_{1}\right), \\
& u_{2}=\left(r \cos \theta_{1},-r \sin \theta_{1}\right),
\end{aligned}
$$

since $u_{0}, u_{1}, u_{2}$ lie on the same circle whose center is $E$ and $\sigma$ is orthogonal to $V\left(u_{1}, u_{2}\right)$ (Figure 2). It follows from $\sigma \cap V\left(u_{1}, u_{2}\right)=\varnothing$ that $\theta_{1}$ must be contained in $[0, \pi / 2)$. Since $E_{\epsilon}=(-\epsilon, 0) \epsilon$ $V\left(u_{1}, u_{2}\right) \backslash V\left(u_{1}, u_{2}, u_{0}\right)$ for small $\epsilon>0$, we have $d\left(E_{\epsilon}, u_{1}\right)<d\left(E_{\epsilon}, u_{0}\right)$ and

$$
\begin{aligned}
0 & <d\left(E_{\epsilon}, u_{0}\right)^{2}-d\left(E_{\epsilon}, u_{1}\right)^{2} \\
& =\left(\left(r \cos \theta_{0}+\epsilon\right)^{2}+\left(r \sin \theta_{0}\right)^{2}\right) \\
& -\left(\left(r \cos \theta_{1}+\epsilon\right)^{2}+\left(r \sin \theta_{1}\right)^{2}\right) \\
& =2 r \epsilon\left(\cos \theta_{0}-\cos \theta_{1}\right) .
\end{aligned}
$$

Hence $-\pi / 2<-\theta_{1}<\theta_{0}<\theta_{1}<\pi / 2$ holds and $\tau$ is an obtuse triangle whose longest edge is $\sigma$. Therefore, for $\sigma^{\prime}=\overline{u_{0} u_{1}}$ and $\sigma^{\prime \prime}=\overline{u_{0} u_{2}}$, we can show that $r_{\sigma^{\prime}}<r_{\tau}$ and $r_{\sigma^{\prime \prime}}<r_{\tau}$ from the general position assumption and the definition of the birth radius of a simplex $\omega$

$$
\begin{gathered}
r_{\omega}=\inf \{\text { the radius of } B \mid B \text { is an empty ball } \\
\text { satisfying } \partial B \supset(\text { vertices of } \omega)\} .
\end{gathered}
$$

Since any other birth radii are different from $r_{\tau}$ by the general position assumption, we have $\operatorname{Alp}\left(P, r_{\tau}+\right) \backslash \operatorname{Alp}\left(P, r_{\tau}\right)=\{\sigma, \tau\}$. Therefore, the inclusion

$$
\left|\left\{\sigma^{\prime}, \sigma^{\prime \prime}, u_{0}, u_{1}, u_{2}\right\}\right| \hookrightarrow\left|\left\{\tau, \sigma, \sigma^{\prime}, \sigma^{\prime \prime}, u_{0}, u_{1}, u_{2},\right\}\right|,
$$

leads to the desired deformation retract.

The case of $L=3, \operatorname{dim} \sigma=1$, and $\operatorname{dim} \tau=2$ can be proved in the same way by considering the plane that contains three vertices of $\tau$. 


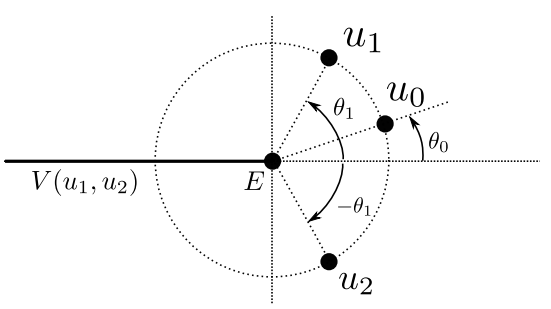

Figure 2: Orthogonal coordinate system for an attaching 2simplex $\tau=\left\{u_{0}, u_{1}, u_{2}\right\}$.

The case of $L=3, \operatorname{dim} \sigma=2$, and $\operatorname{dim} \tau=3$ can also be proved in a similar way by replacing $V\left(u_{1}, u_{2}\right)$ in the above argument to $V\left(u_{1}, u_{2}, u_{3}\right)$. In this case, the 3 -simplex $\tau$ is given by a tetrahedron whose four vertices are on a half side of the circumsphere of the tetrahedron.

The following corollary is a straightforward consequence of Lemma 4.4 .

Corollary 4.5. For a simplex $\tau$, if $r_{\tau}$ is either a birth time or a death time in the persistence diagram, $\tau$ is an attaching simplex and does not attach any faces of $\tau$.

Proof. (Proposition 4.3 Since $d$ is a death time in $D_{\ell}(\operatorname{Alp}(P))$, an $(\ell+1)$-simplex $\tau$ is born at time $d$, hence $d=r_{\tau}$. From Corollary 4.5, $\tau$ is an attaching simplex and does not attach any faces of $\tau$. With the general position assumption, $\tau$ is the unique simplex satisfying $d=r_{\tau}$.

Next, we consider the change at time $b$. Since the birth radius of any simplex is not contained in $(b, d)$ and $\tau$ is unique as above, $\operatorname{Alp}(P, \alpha)=$ $\operatorname{Alp}\left(P, r_{\tau^{+}}\right) \backslash\{\tau\}$ for any $\alpha \in(b, d]$. Therefore, one of the $\ell$-dimensional faces $\sigma$ of $\tau$ appears at time $b$. Otherwise, the generator by $\partial \tau$ keeps unchanged at time $b$ and this contradicts the fact that $b$ is the birth time of the pair $(b, d)$. The simplex $\sigma$ is an attaching simplex from Corollary 4.5, and hence this concludes the proof.

Now, we show that if a birth-death pair $(b, d)$ is close enough $b \approx d$ for the birth radius of any simplex not to be contained in $(b, d)$, then a singular value close to zero appears in the derivative of the persistence map. In this case, from Proposition 4.3. there exist an $(\ell+1)$-simplex $\tau$ and its face $\sigma$ satisfying $r_{\tau}=d$ and $r_{\sigma}=b$. Let $p_{1}, \ldots, p_{\ell}$ be the vertices of $\sigma$ and $p_{0}, p_{1}, \ldots, p_{\ell}$ be the vertices of $\tau$. Let $c_{0}$ be the center of the minimal circumsphere of $\tau$ and $c_{1}$ be the center of the minimal circumsphere of $\sigma$.
Let $r$ be the length of $\overline{p_{0} c_{0}}$, the radius of minimal circumsphere of $\tau$, and $\theta$ be the angle of $\angle c_{0} p_{1} c_{1}$ (Figure 3. From the fact 3 of the Voronoi decomposition, $\overline{c_{0} c_{1}}$ is orthogonal to $\sigma$. Therefore we can represent $r_{\tau}$ and $r_{\sigma}$ as follows:

$$
\begin{aligned}
& r_{\tau}=r, \\
& r_{\sigma}=r \cos \theta .
\end{aligned}
$$
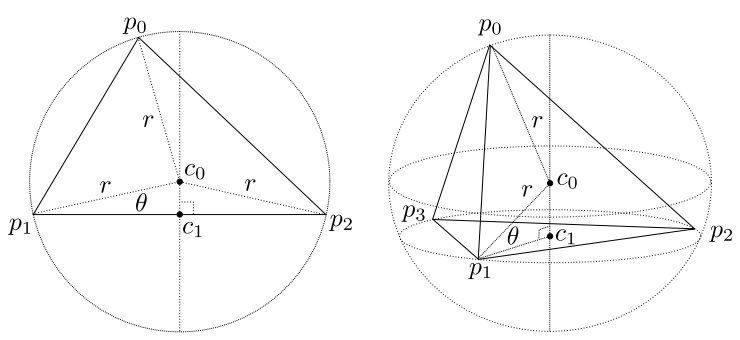

Figure 3: In the left figure, $c_{0}$ is the center of the circumcircle of the triangle $\left\{p_{0}, p_{1}, p_{2}\right\}, c_{1}$ is the center of $\overline{p_{1} p_{2}}, r$ is the radius of the circumcircle, and $\theta$ is $\angle c_{0} p_{1} c_{1}$. In the right figure, $c_{0}$ is the center of the circumsphere of the tetrahedron $\left\{p_{0}, p_{1}, p_{2}, p_{3}\right\}, c_{1}$ it the center of the circumcircle of the triangle $\left\{p_{1}, p_{2}, p_{3}\right\}, r$ is the radius of the circumsphere, and $\theta$ is $\angle c_{0} p_{1} c_{1}$.

We consider a map $f: U \rightarrow \mathbb{R}^{2}$ given by

$$
f: u \mapsto\left[\begin{array}{l}
r_{\tau} \\
r_{\sigma}
\end{array}\right]
$$

assigning one birth-death pair. This map is one component of the persistence map and can be decomposed as follows

$$
u \stackrel{f_{1}}{\longrightarrow}(r, \theta) \stackrel{f_{2}}{\longrightarrow}\left(r_{\tau}, r_{\sigma}\right)
$$

We can easily show the following facts:

- $r_{\tau}=r_{\sigma}$ if and only if $\theta=0$.

- If $\theta=0$,

$$
D f_{2}=\left[\begin{array}{ll}
1 & 0 \\
1 & 0
\end{array}\right]
$$

holds.

From these facts, we have $\theta \approx 0$ for $r_{\tau} \approx r_{\sigma}$ and

$$
D f_{2} \approx\left[\begin{array}{ll}
1 & 0 \\
1 & 0
\end{array}\right] \text {. }
$$

Since the matrix of the right hand side is not surjective, $D f=D f_{2} \circ D f_{1}$ has a singular value close to zero, and hence the derivative of the persistence map has a singular value close to zero. 


\subsubsection{For the case of Vietoris-Rips filtrations}

For the case of Vietoris-Rips filtrations, a birthdeath pair $(b, d)$ with $b \approx d$ does not necessarily imply the existence of a singular value close to zero. We show such an example for a point cloud $P=$ $\{A, B, C, D\}$ in $\mathbb{R}^{2}$ and $D_{1}(\operatorname{VR}(P))$. We assume the followings:

- $|\overline{A B}|,|\overline{B C}|,|\overline{C D}|<|\overline{A D}|<|\overline{B D}|<|\overline{A C}|$.

- $|\overline{A D}| \approx|\overline{B D}|$.

From the assumption, the triangle $A B D$ is close to an isosceles triangle. The persistence diagram $D_{1}(\operatorname{VR}(P))$ has a unique birth-death pair $(b, d)$ where $b=|\overline{A D}|$ and $d=|\overline{B D}|$ (Figure 4).

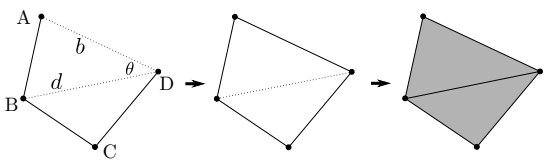

Figure 4: A point cloud with four points. $D_{1}(\operatorname{VR}(P))$ has a unique birth-death pair $(b, d)$

The persistence map $f$ is

$$
f=\left[\begin{array}{l}
b \\
d
\end{array}\right],
$$

and from the computation in Lemma 3.6. $D f$. $(D f)^{T}$ is

$$
D f \cdot(D f)^{T}=\left[\begin{array}{cc}
2 & \cos \theta \\
\cos \theta & 2
\end{array}\right]
$$

where $\theta=\angle A D B$. Since eigenvalues of $D f \cdot(D f)^{T}$ are squares of singular values of $D f$, the singular values are $\sqrt{2 \pm \cos \theta}$ and are away from zero, although $b \approx d$.

\section{Computations}

In this section, we present some numerical examples of continuations of point clouds using persistence diagrams. Alpha filtrations are used for all examples, and the coordinate system described in Section 4.4 is adopted to eliminate the translation and rotation symmetries.

Example 5.1 (Deformation of a tetrahedron). As a first example, we consider alpha filtrations constructed from four points in $\mathbb{R}^{3}$ (a tetrahedron) and apply our continuation algorithm to $D_{2}$. We take as the initial point cloud $P=\left\{u_{0}, u_{1}, u_{2}, u_{3}\right\}$, where $u_{0}=(0,0,0), u_{1}=(8,0,0), u_{2}=(5,6,0)$, and $u_{3}=(4,2,6)$. The 2 nd persistence diagram of $P$ is $D_{2}=\{(4.42719,4.59015)\}$. From this initial data, we try to deform $D_{2}$ to the target persistence data $\{(8.42719,8.89015)\}$ using our continuation method. Due to the coordinate system adopted to eliminate symmetries, 4.6, we have that the degree of freedom of the point cloud is six, and the persistence map can be expressed as $f: U \subset \mathbb{R}^{6} \rightarrow$ $\mathbb{R}^{2}$. For this example we used $\|\Delta v\|=0.01$ as the step size in the continuation, and $\epsilon=\mathrm{d}^{1}$.

Figures 5 and 6 show the point clouds and the persistence diagrams during the continuation process. In this computation we successfully reached the target persistence diagram $\{(8.42719,8.89015)\}$. However notice that there seems to be a non-smooth point on the blue curves given by the point clouds during continuation in Figure 5. To try to understand this event, we look at the birth radii of the 2-simplices during the continuation process (Figure 7). Notice that at some point during the continuation process, two of the birth radii coincide, hence breaking the second condition of the alpha general position assumption (Definition 2.2). This point corresponds exactly to the non-smooth point in Figure 7. This is a point where the derivative $D f$ is not uniquely defined, hence the continuation curve is not smooth there. The fact that we are performing the continuation numerically, and hence the birth radii are very close but not exactly equal, makes it possible to go forward with the continuation process. At each step of the continuation the birth radius that is slightly larger is used to compute $D f$, and the 2-simplex corresponding to this radius can change at each continuation step. We can see this from the singular values of $D f$ show in Figure 8

Example 5.2 (Image of the persistence map). In this example we use the same point cloud (tetrahedron) as in Example 5.1 to explore the image of the persistence map. For a tetrahedron, the image of the persistence map is the strip region between the diagonal and the line of persistence diagrams of regular tetrahedrons (see Figure 9 and Theorem 6.1 in the Appendix for a proof of the $D_{1}$ case). In this example we used $\|\Delta v\|=0.001$ and $\epsilon=0$.

\footnotetext{
${ }^{1}$ The 2-dimensional diagram $D_{2}$ of a tetrahedron has at most one birth-death pair, hence we cannot cut off points close to the diagonal and therefore we use $\epsilon=0$.
} 


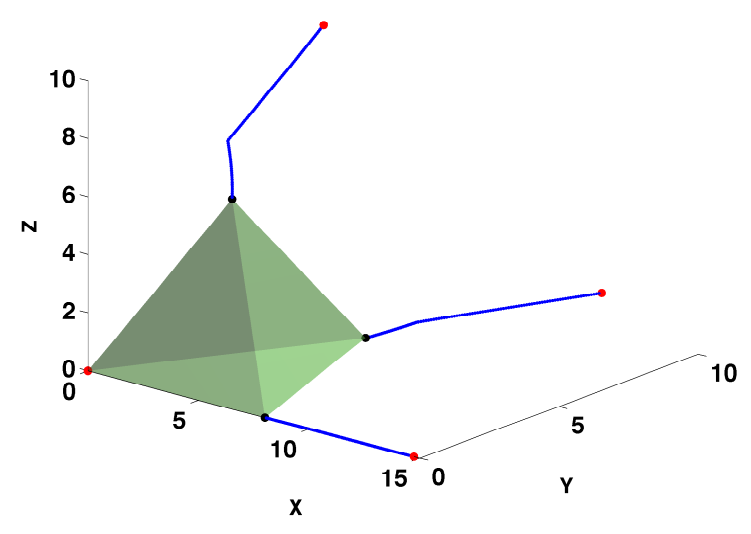

Figure 5: Deformation of the point cloud during continuation. The black dots are the initial point cloud and the red dots are the final one. The blue curves show the movement of the points during the continuation. Note that the first vertex is fixed at the origin and the second one is only allowed to move along the $x$-axis.

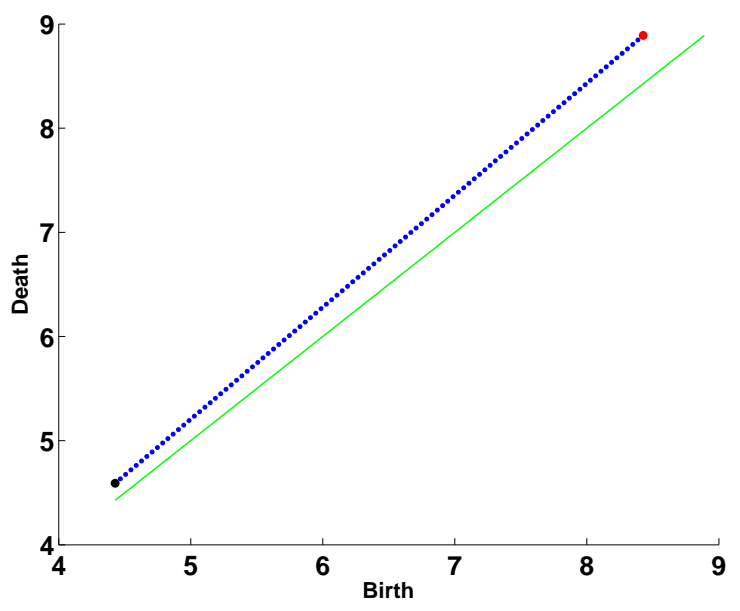

Figure 6: Persistence diagrams $D_{2}$ during the continuation process. The black dot represents the initial persistence diagram and the red dot represents the target diagram. The blue dots represent the persistence diagrams during the continuation process.

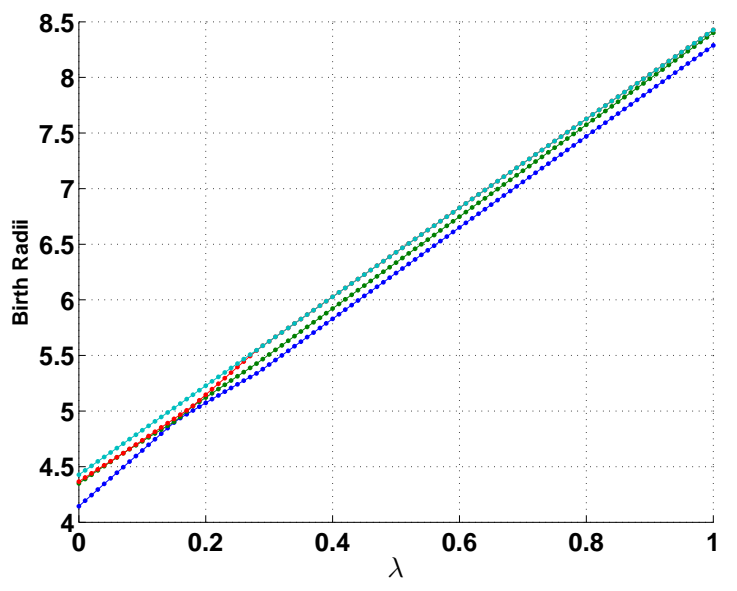

Figure 7: Birth radii of the 2-simplices along the continuation curves in Figure 5

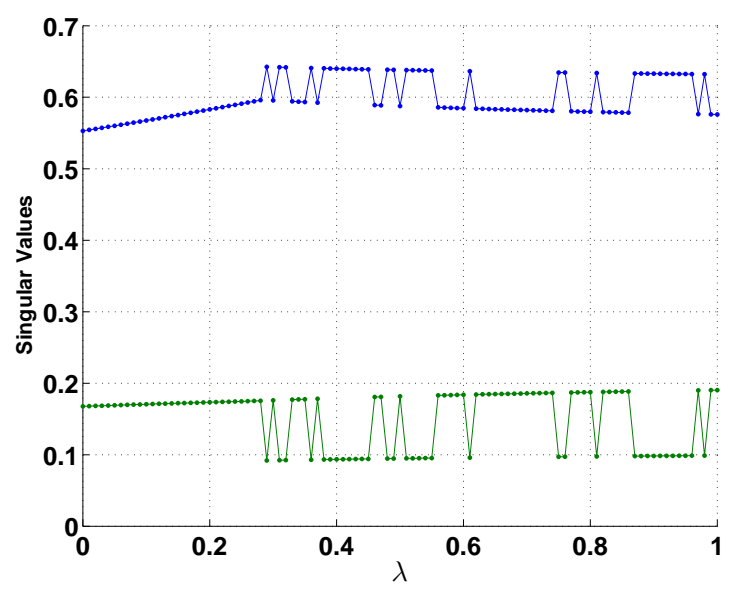

Figure 8: Singular values of the derivative $D f$ along the continuation in Figure 5 
From the initial persistence diagram $D_{2}=$ $\{(4.42719,4.59015)\}$ we try to continue to the target persistence $\{(6.42719,7.09015)\}$, which is outside of the image of the persistence map. Hence, as expected, in this case we fail to reach the target persistence and can only continue up to the boundary of the image, as we can see in Figure 9. As we approach the boundary of the image, the method fails because the number of Newton iterations needed for convergence increases dramatically as is shown in Figure 10. In the last steps of the continuation the birth radii of the 1-simplices are very similar and the birth radii of the 2-simplices are all virtually the same as we can see in Figures 11 and 12. hence confirming that we have continued to a regular tetrahedron. Notice also from Figures 11 and 12 that two or more birth radii are equal during the continuation, hence the general position assumption (Definition 2.2 is violated. However, as noted in Example 5.1, the continuation method still works as long as we are within the image of the persistence map.

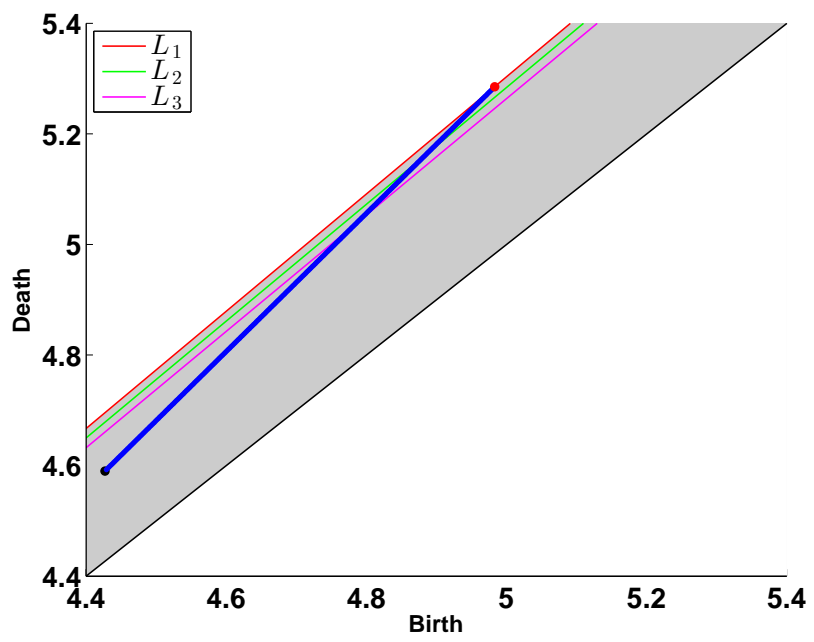

Figure 9: The image of the persistence map of $D_{2}$ for one tetrahedron (four points in $\mathbb{R}^{3}$ ) is the shaded region between the diagonal and the line $L_{1}$ of the persistence diagrams of regular tetrahedrons (obtained by similarity deformations). The lines $L_{2}$ and $L_{3}$ correspond to the persistence diagrams of tetrahedrons with three and two congruent faces, respectively. The blue line shows the continuation of the initial point cloud all the way to the boundary of the image.

Example $\quad \mathbf{5 . 3}$ (Towards the diagonal). In this example we take the point cloud $P=\left\{u_{0}, u_{1}, u_{2}, u_{3}\right\}$, where $u_{0}=(0,0,0)$, $u_{1}=(9.991,0,0), u_{2}=(4.9955,8.65246,0)$, and $u_{3}=(4.9955,2.88415,8.15762)$, which represents

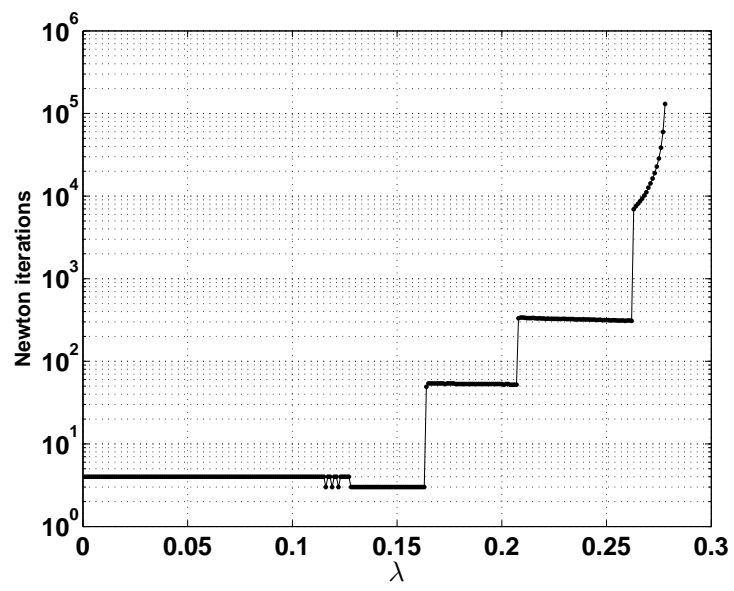

Figure 10: Number Newton iterations during each step of the continuation in Example 5.2

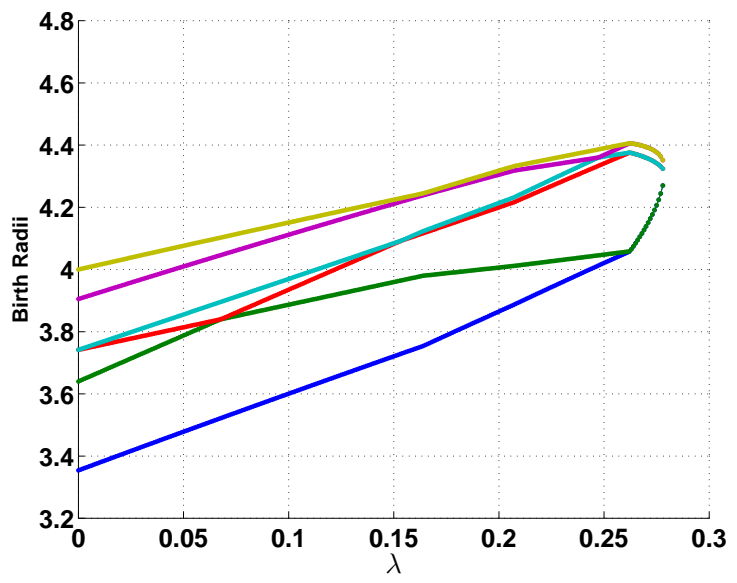

Figure 11: Birth radii of the 1-simplices along the continuation in Example 5.2

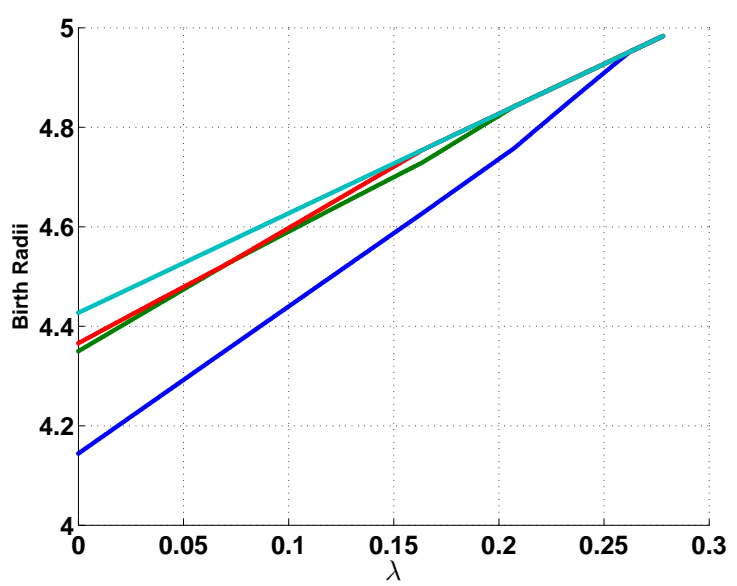

Figure 12: Birth radii of the 2-simplices along the continuation in Example 5.2 
a nearly regular tetrahedron, and try to continue the persistence diagram $D_{2}$ towards the diagonal. From the discussion in Section 4.5 this will lead to singular values close to zero. The 2 nd persistence diagram of $P$ is $D_{2}=\{(5.76831,6.11821)\}$, and the target persistence diagram is set to be $\{(5.94841,5.94841)\}$ which is on the diagonal. In the computations we used $\|\Delta v\|=0.001$ and $\epsilon=0$.

In this case the computations work well all the way to a point nearly on the diagonal. In Figure 13 we show the persistence diagrams along the continuation. The singular values of the derivative are shown in Figure 14. As expected, one of the singular values approaches zero towards the end of the continuation. However, in spit of this, the continuation works all the way to a point essentially on the diagonal. Note that we cannot continue to a point exactly on the diagonal, since the persistence diagram would be empty in that case. However the persistence diagram that we arrive at the end of the continuation in this example is only "numerically" on the diagonal, that is, it is on the diagonal up to the error tolerance of the Newton-Raphson method. As described in Section 4.2 the method will fail if we try to continue to a point exactly on the diagonal, since in that case we would have a zero singular value.

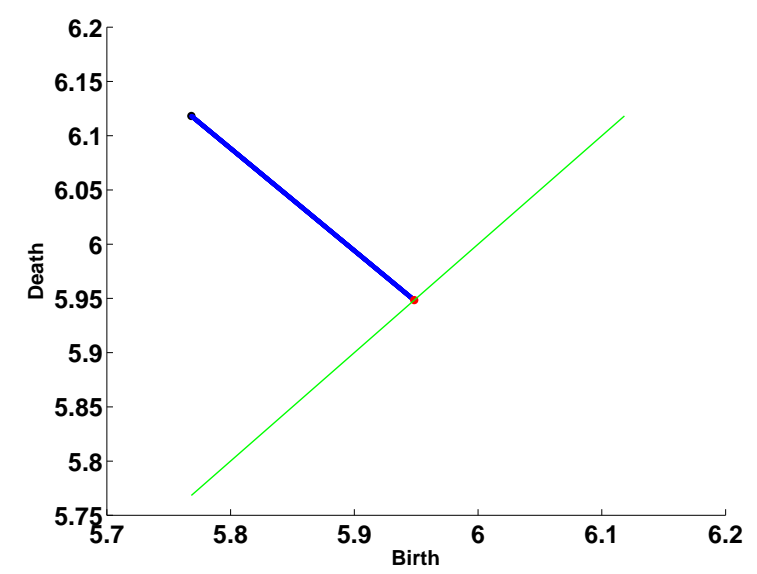

Figure 13: Persistence diagrams $D_{2}$ during the continuation process. The black dot represents the initial persistence diagram and the red dot represents the final diagram. Notice that the final diagram is "numerically" on the diagonal.

Example 5.4 (Continuation of $D_{1}$ ). In this example we take the point cloud data $P=$ $\left\{u_{0}, u_{1}, u_{2}, u_{3}\right\}$, where $u_{0}=(0,0,0), u_{1}=(1,0,0)$, $u_{2}=(1.1,1.2,0)$, and $u_{3}=(0.5,0.6,1.3)$, and try to continue the initial persistence diagram

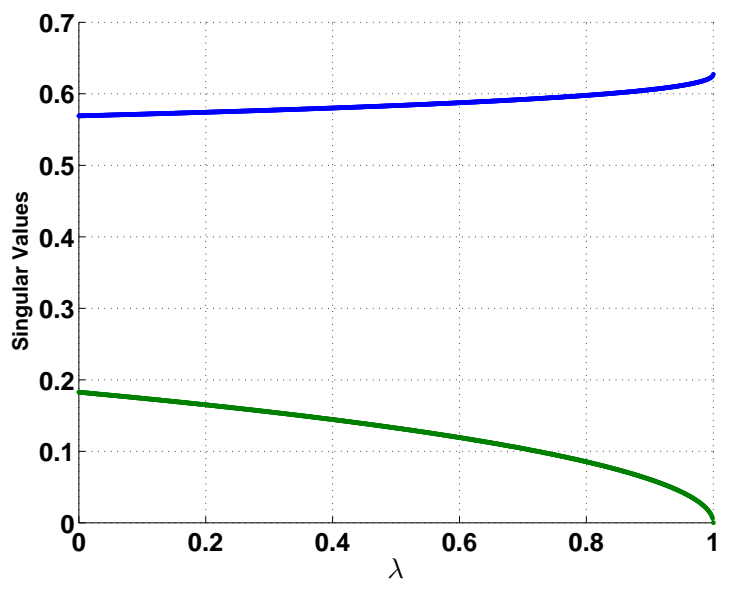

Figure 14: Singular values of the derivative $D f$ along the continuation in Example 5.3. Notice that one of the singular values approach zero as we reach the end of the continuation.

$D_{1}=\{(0.758288,0.803195),(0.776209,0.834393)\}$ to the target 1-dimensional persistence diagram $\{(0.770801,0.817236),(0.798346,0.863075)\} . \quad$ In these computations we used $\|\Delta v\|=0.001$ and $\epsilon=0$. There were only two points in the persistence diagram during all the steps of the continuation, hence the choice $\epsilon=0$. The computations worked well all the way to the target persistence. In Figure 15 we show the point cloud and the persistence diagrams along the continuation.

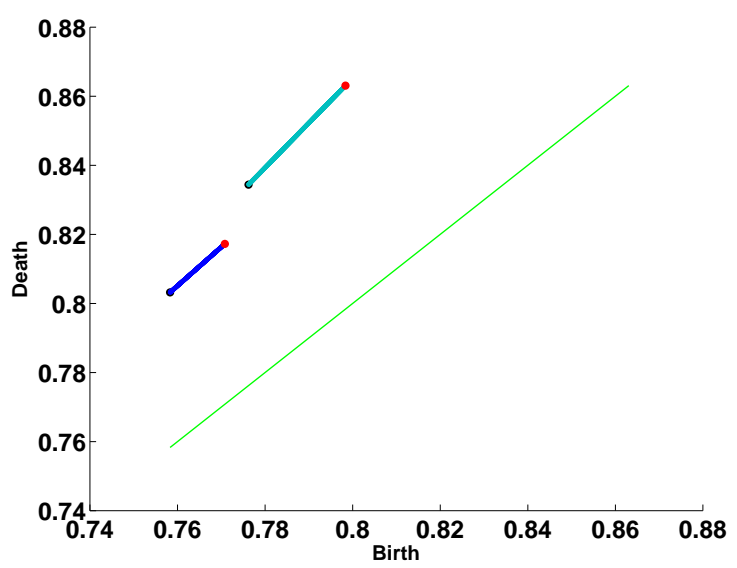

Figure 15: Persistence diagrams $D_{1}$ during the continuation process. The black dots represent the initial persistence diagram and the red dots represent the final diagram.

Example 5.5 (Deformation of a dodecahedron). In this example we take as the initial point cloud the vertices of a regular dodecahedron and try to apply our continuation method to increase both the 
birth and the death radii of the $D_{2}$ generator. In these computations we used $\|\Delta v\|=0.01$ and $\epsilon=$ $10^{-3}$. The continuation works all the way to the target persistence diagram. Figure 16 shows the deformation of the point cloud and Figure 17 shows the diagrams along the continuation.

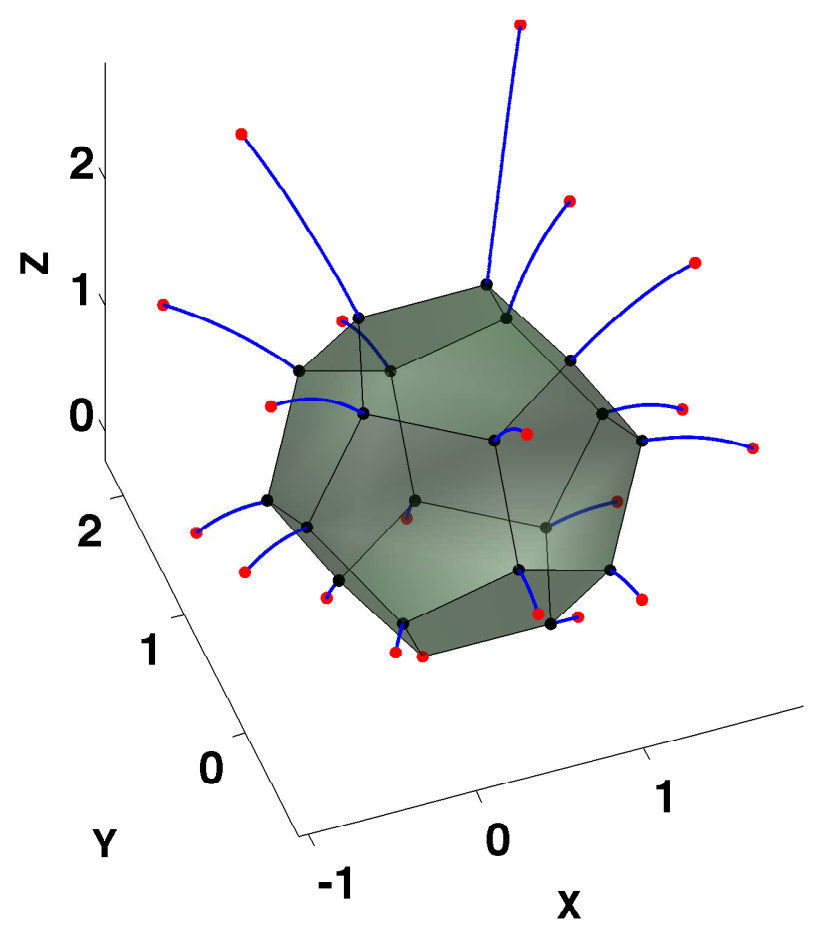

Figure 16: Deformation of the point cloud during continuation. The black dots are the initial point cloud and the red dots are the final one. The blue curves show the movement of the points during the continuation.

Example 5.6 (Deformation of a sphere). In this example we take as the initial point cloud 100 uniform point on a sphere and try to apply our continuation method to the largest generator of $D_{2}$. In these computations we used $\|\Delta v\|=0.03$ and $\epsilon=10^{-5}$. The continuation works all the way to the target persistence diagram. Figure 18 shows the deformation of the point cloud and Figure 19 shows the diagrams along the continuation.

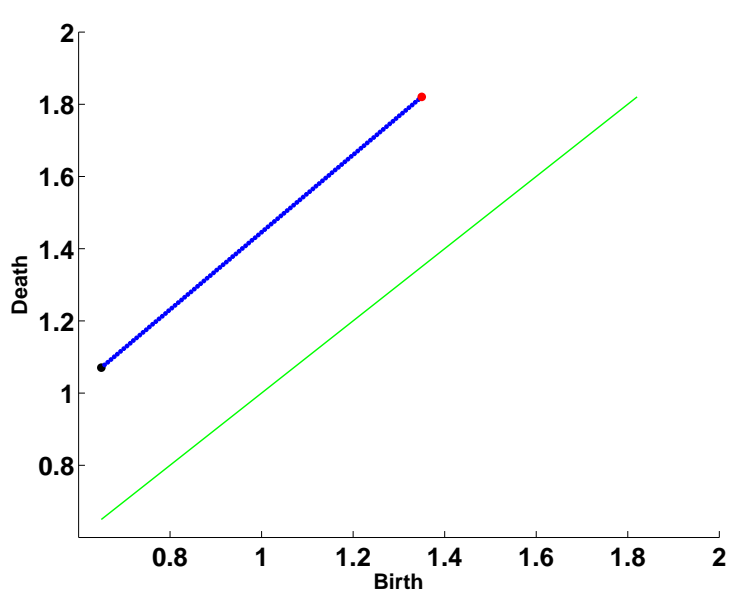

Figure 17: Persistence diagrams $D_{2}$ during the continuation process. The black dot represents the initial persistence diagram and the red dot represents the final diagram.

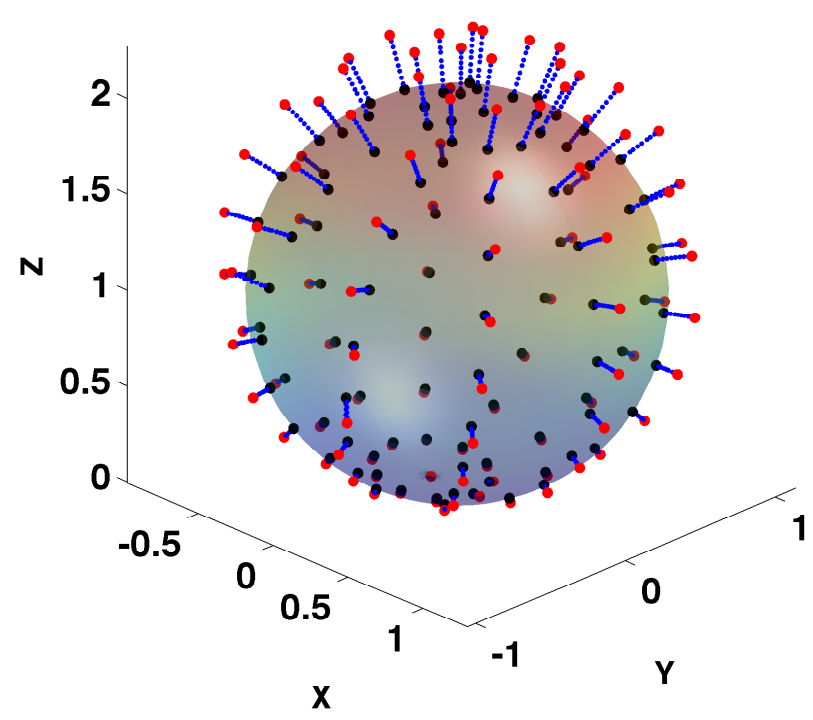

Figure 18: Deformation of the point cloud during continuation. The black dots are the initial point cloud and the red dots are the final one. The blue dots show the movement of the points during the continuation. 


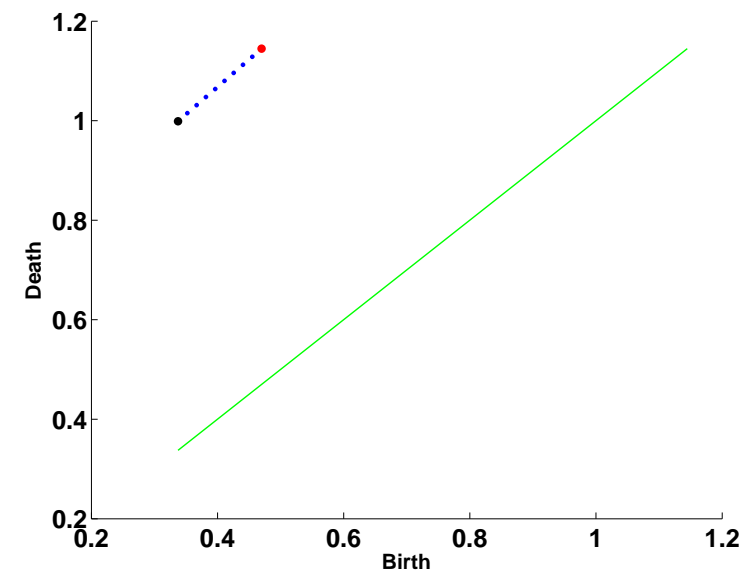

Figure 19: Persistence diagrams $D_{2}$ during the continuation process. The black dot represents the initial persistence diagram and the red dot represents the final diagram.

\section{Conclusion}

In this paper, we have studied the continuation of point clouds by persistence diagrams. In the following, we list some future improvement of our method.

1. In the presented method, we have treated persistence diagrams in an Euclidean space whose dimension is determined by the input persistence diagram. This vectorization is simple and describes the essential part for the continuation method. However, it does not allow to change the number of generators in the persistence diagrams during the continuation because of the fixed dimension of the Euclidean space. To overcome this restriction, it can be useful to vectorize the persistence diagrams into a bigger space and construct a similar continuation method. The space of persistence landscape 24 or a vectorization using kernel methods 25] should be considered as possible candidates.

2. Our algorithm for computing persistence diagrams in this paper is not sophisticated, and hence, there is room for improvement. Standard reduction methods such as [26] can be implemented and will reduce the computational cost. Furthermore, since the changes in the point clouds at each step of the continuation is supposed to be small, the vineyard algorithm 27. can effectively work for fast computations.

\section{Acknowledgments}

The authors would like to thank Shouhei Honda for useful discussions. M. G. was partially supported by FAPESP grants 2013/07460-7 and 2010/00875-9, and by CNPq grant 306453/2009-6, Brazil. Y. H. and I. O. were partially supported by JSPS Grant-in-Aid (24684007, 26610042).

\section{Appendix. Image of the Persistence Map of $D_{1}$ for a triangle}

Theorem 6.1. If a point cloud $P$ has only three points, $D_{1}(\operatorname{Alp}(P))$ has at most one birth-death pair. If $D_{1}(\operatorname{Alp}(P))$ has such a pair $(b, d)$, the ratio $d / b$ is smaller than or equal to $2 / \sqrt{3}$. Moreover, $d / b=2 / \sqrt{3}$ if and only if the triangle is regular.

Proof. From the basic properties of alpha filtrations, $D_{1}(\operatorname{Alp}(P))=\{(b, d)\}$ if and only if the triangle is acute. If not, $D_{1}(\operatorname{Alp}(P))$ is empty. Hence we assume that the triangle is acute.

Let $p_{0}, p_{1}, p_{2}$ be the three vertices of the triangle, $c$ be the center of the circumcircle, $r$ be the radius of the circumcircle, and $\theta_{0}, \theta_{1}, \theta_{2}$ be $\angle c p_{1} p_{2}, \angle c p_{2} p_{0}$, and $\angle c p_{0} p_{1}$, respectively (Figure 20).

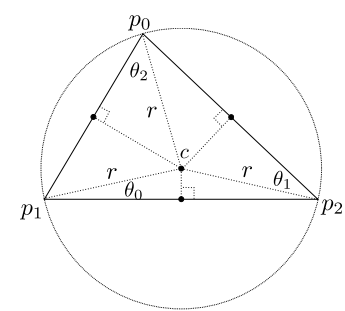

Figure 20: The relation of the circumcircle and internal angles of the triangle $\left\{p_{0}, p_{1}, p_{2}\right\}$

Hence, $\left|p_{1} p_{2}\right|=2 r \cos \theta_{0},\left|p_{2} p_{0}\right|=2 r \cos \theta_{1},\left|p_{0} p_{1}\right|=$ $2 r \cos \theta_{2}$ and the birth time $b$ is

$$
b=\max \left\{r \cos \theta_{0}, r \cos \theta_{1}, r \cos \theta_{2}\right\},
$$

and the death time $d$ is $r$. The ratio of $d / b$ is

$$
\begin{aligned}
& r / \max \left\{r \cos \theta_{0}, r \cos \theta_{1}, r \cos \theta_{2}\right\} \\
= & \left(\max \left\{\cos \theta_{0}, \cos \theta_{1}, \cos \theta_{2}\right\}\right)^{-1} .
\end{aligned}
$$

Hence the problem is minimizing

$$
\max \left\{\cos \theta_{0}, \cos \theta_{1}, \cos \theta_{2}\right\}
$$


subject to

$\theta_{0}, \theta_{1}, \theta_{2}>0$ (the triangle is acute) and

$\theta_{0}+\theta_{1}+\theta_{2}=\pi / 2$ (sum of the internal angles).

Since $\cos \theta$ is monotonely decreasing on the interval $[0, \pi / 2]$, a minimum attains at $\theta_{0}=\theta_{1}=\theta_{2}=\pi / 6$ and the minimum is $\cos (\pi / 6)=\sqrt{3} / 2$.

\section{References}

\section{References}

[1] G. Carlsson. Topology and Data. Bull. Amer. Math. Soc. 46 (2009), 255-308.

[2] H. Edelsbrunner and J. Harer. Computational Topology: An Introduction. AMS (2010).

[3] H. Edelsbrunner, D. Letscher, and A. Zomorodian. Topological Persistence and Simplification. Discrete Comput. Geom. 28(4) (2002), 511-533.

[4] A. Zomorodian and G. Carlsson. Computing Persistent Homology. Discrete Comput. Geom. 33(2) (2005), 249 274.

[5] T. Nakamura, Y. Hiraoka, A. Hirata, E. G. Escolar, K. Matsue, and Y. Nishiura. Description of medium-range order in amorphous structures by persistent homology. arXiv:1501.03611

[6] T. Nakamura, Y. Hiraoka, A. Hirata, E. G. Escolar, and Y. Nishiura. Persistent homology and many-body atomic structure for medium-range order in the glass. Accepted in Nanotechnology.

[7] M. Gameiro, Y. Hiraoka, S. Izumi, M. Kramar, K. Mischaikow, and V. Nanda. Topological measurement of protein compressibility via persistent diagrams. Japan J. Indust. Appl. Math. 32 (2015), 1-17.

[8] V. de Silva and R. Ghrist. Coverage in sensor networks via persistent homology. Algebraic and Geometric Topology 7 (2007), 339-358.

[9] E. L. Allgower and K. Georg. Introduction to Numerical Continuation Methods. SIAM (2003).

10] B. Krauskopf, H. Osinga, and J. Galán-Vioque (Eds.). Numerical Continuation Methods for Dynamical Systems: Path following and boundary value problems. Springer (2007).

[11] H. Edelsbrunner and E. Mücke. Three-Dimensional Alpha Shapes. ACM Transactions on Graphics 13(1) (1994), 43-72.

[12] CGAL. Computational Geometry Algorithms Library. http://www.cgal.org/

13] F. Chazal, V. de Silva, and S. Oudot. Persistence Stability for Geometric Complexes. Geometriae Dedicata 173(1) (2014), 193-214.

[14] J. D. Boissonnat, R. Dyer, and A. Ghosh. The Stability of Delaunay Triangulations. INRIA Research Report No. 8276 (2013)

[15] U. Bauer, M. Kerber, and J. Reininghaus. Clear and Compress: Computing Persistent Homology in Chunks. In Topological Methods in Data Analysis and Visualization III: Theory, Algorithms, and Applications. Springer (2014), 103-117.

[16] H. Federer. Geometric measure theory, Springer-Verlag, 1996.
[17] J. M. Ortega and W. C. Rheinboldt. Iterative Solution of Nonlinear Equations in Several Variables. SIAM (2000).

[18] A. Ben-Israel and T. Greville. Generalized Inverses: Theory and Applications, 2nd ed. . Springer (2003).

[19] R. A. Horn and C. R. Johnson. Matrix analysis. Cambridge University Press (1990).

[20] A. Ben-Israel. A Newton-Raphson Method for the Solution of Systems of Equations. J. Math. Anal. and Appl. 15 (1966), 243-252.

[21] A. Ben-Israel. A Modified Newton-Raphson Method for the Solution of Systems of Equations. Israel J. Math. 3 (1965), 94-98.

[22] M. de Berg, O. Cheong, M. van Kreveld, and M. Overmars. Computational Geometry: Algorithms and Applications. Springer (2008).

[23] http://www.wpi-aimr.tohoku.ac.jp/hiraoka_labo/ continuation-paper/

[24] P. Bubenik. Statistical Topological Data Analysis using Persistence Landscapes. Journal of Machine Learning Research 16 (2015), 77-102.

[25] J. Reininghaus, S. Huber, U. Bauer, and R. Kwitt. A Stable Multi-Scale Kernel for Topological Machine Learning. Accepted in the IEEE Conference on Computer Vision and Pattern Recognition (CVPR) 2015.

[26] K. Mischaikow and V. Nanda. Morse Theory for Filtrations and Efficient Computation of Persistent Homology. Discrete Comput. Geom. 50 (2013), 330-353.

[27] D. Cohen-Steiner, H. Edelsbrunner, and D. Morozov. Vines and Vineyards by Updating Persistence in Linear Time. Proceedings of the twenty-second annual symposium on Computational geometry (2006), 119-126. 